\title{
Simulation of inertial fibre orientation in turbulent flow
}

Cite as: Phys. Fluids 28, 063307 (2016); https://doi.org/10.1063/1.4954214

Submitted: 13 January 2016 . Accepted: 04 June 2016 . Published Online: 21 June 2016

Derrick O. Njobuenwu (D), and Michael Fairweather

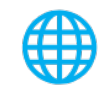

View Online
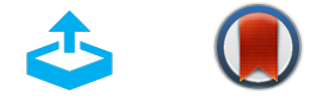

Export Citation

CrossMark

\section{ARTICLES YOU MAY BE INTERESTED IN}

Orientation, distribution, and deposition of elongated, inertial fibers in turbulent channel flow

Physics of Fluids 22, 033301 (2010); https://doi.org/10.1063/1.3328874

On the relative rotational motion between rigid fibers and fluid in turbulent channel flow

Physics of Fluids 28, 013301 (2016); https://doi.org/10.1063/1.4937757

Four-way coupled simulations of small particles in turbulent channel flow: The effects of particle shape and Stokes number

Physics of Fluids 27, 083301 (2015); https://doi.org/10.1063/1.4927277

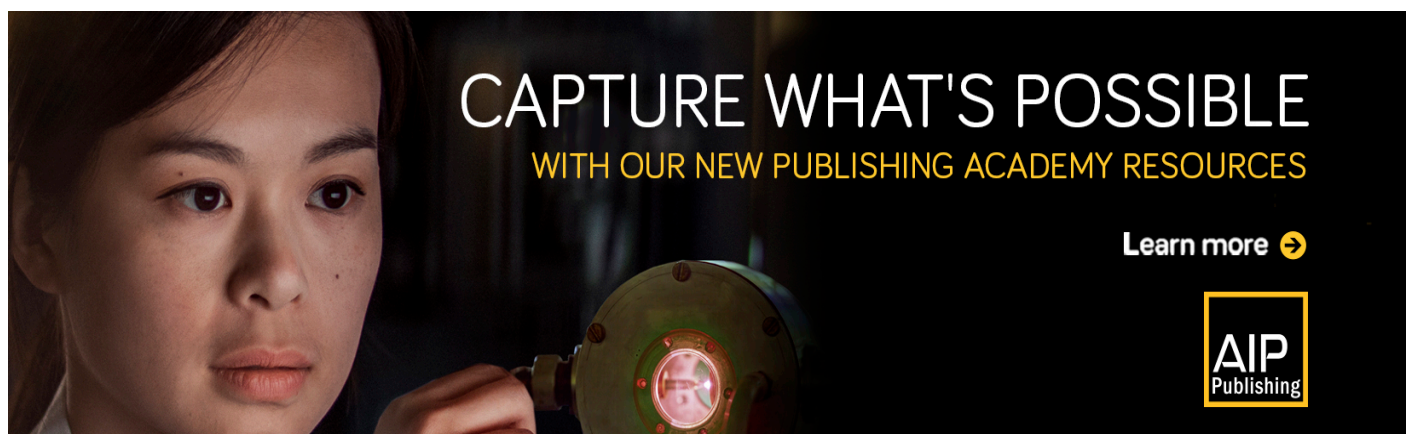




\title{
Simulation of inertial fibre orientation in turbulent flow
}

\author{
Derrick O. Njobuenwu and Michael Fairweather \\ School of Chemical and Process Engineering, University of Leeds, \\ Leeds LS2 9JT, United Kingdom
}

(Received 13 January 2016; accepted 4 June 2016; published online 21 June 2016)

The spatial and orientational behaviour of fibres within a suspension influences the rheological and mechanical properties of that suspension. An Eulerian-Lagrangian framework to simulate the behaviour of fibres in turbulent flows is presented. The framework is intended for use in simulations of non-spherical particles with high Reynolds numbers, beyond the Stokesian regime, and is a computationally efficient alternative to existing Stokesian models for fibre suspensions in turbulent flow. It is based on modifying available empirical drag correlations for the translation of non-spherical particles to be orientation dependent, accounting for the departure in shape from a sphere. The orientational dynamics of a particle is based on the framework of quaternions, while its rotational dynamics is obtained from the solution of the Euler equation of rotation subject to external torques on the particle. The fluid velocity and turbulence quantities are obtained using a very high-resolution large eddy simulation with dynamic calibration of the sub-grid scale energy containing fluid motions. The simulation matrix consists of four different fibre Stokes numbers $(S t=1,5,25$, and 125) and five different fibre aspect ratios $(\lambda=1.001,3,10,30$, and 50), with results considered at four distances from a channel wall (in the viscous sub-layer, buffer, and fully turbulent regions), which are taken as a measure of the flow velocity gradient, all at a constant fibre to fluid density ratio $\left(\rho_{p} / \rho=760\right)$ and shear Reynolds number $R e_{\tau}=150$. The simulated fibre orientation, concentration, and streakiness confirm previous experimentally observed characteristics of fibre behaviour in turbulence, and that of direct numerical simulations of fibres in Stokesian, or creeping flow, regimes. The fibres exhibit translational motion similar to spheres, where they tend to accumulate in the near-wall (viscous sub-layer and buffer) region and preferentially concentrate in regions of low-speed streaks. The current results further demonstrate that the fibres' translational dynamics, in terms of preferential concentration, is strongly dependent on their inertia and less so on their aspect ratio. However, the contrary is the case for the fibre alignment distribution as this is strongly dependent on the fibre aspect ratio and velocity gradient, and only moderately dependent on particle inertia. The fibre alignment with the flow direction is found to be mostly anisotropic where the velocity gradient is large (i.e., viscous sub-layer and buffer regions), but is virtually non-existent and isotropic where the turbulence is near-isotropic (i.e., channel centre). The present investigation highlights that the level of fibre alignment with the flow direction reduces as a fibre's inertia decreases, and as the shape of the fibre approaches that of a sphere. Short fibres, and especially near-spherical $\lambda=1.001$ particles, are found to exhibit isotropic orientation with respect to all directions, whilst sufficiently long fibres align themselves parallel to the flow direction, and orthogonal to the other two co-ordinate directions, and the vorticity and flow velocity gradient directions. Published by AIP Publishing. [http://dx.doi.org/10.1063/1.4954214]

\section{INTRODUCTION}

The dynamics of solid particles suspended in a turbulent channel flow is important in order to understand the hydrodynamics of solid suspensions. Of particular interest here is prediction of the 
flow-induced alignment of non-spherical particles in suspension which is important in many applications, including the kinematics of particles suspended in aquatic media (e.g., micro-organisms, plankton, and engineered nanomaterials), ${ }^{1,2}$ industrial processes such as paper-making, ${ }^{3}$ and the motion of corrosion-related unidentified deposits (CRUD) on the outside of nuclear reactor fuel pins. ${ }^{4}$ Other applications are in the biomedical industries, such as the dispersion of nano-fibres in the respiratory tract and the separation of bio-particles (rod-shaped bacteria and disc-shaped red blood cells) from plasma, and natural phenomena such as ice crystal formation in clouds. ${ }^{5}$

In this work, consideration is limited to rigid fibres, which are very slender bodies and can be treated as high aspect ratio rods, cylinders, needles, or prolate ellipsoids. The aspect ratio, $\lambda=c / a$, defined as the ratio of the length of the semi-major axis, $c$, and the semi-minor axis, $a$, is a key parameter used to characterise such fibres. For high aspect ratio fibres, any suspension generally exhibits anisotropic properties which deviate strongly from those of spherical particles of equal equivalent volume. The interaction of such fibres with their surroundings (fibre-fluid, fibre-fibre, and fibre-wall) all influence the fibres' local concentration and orientation trends; hence, there has been increasing interest in fibre suspensions in recent years. ${ }^{6}$ Most research on non-spherical particle suspension flows focuses on two main aspects. ${ }^{7}$ The first is the translational and rotational motions of the ellipsoidal particles in turbulent flows, for example. ${ }^{8}$ The other is concerned with the modification of flow properties caused by the addition of the particles and, in particular, how the particles alter the fluid's rheological properties, for example. ${ }^{9}$ Other research on this latter topic has found that fibres can induce significant drag reduction and modify the velocity fluctuations in the fluid, with enhancement in the streamwise and a reduction in the wall-normal and spanwise directions. ${ }^{10,11}$ Our interest is in the former area, since the fibre orientation influences the mechanical, thermal, rheological, and electrical properties of a suspension. ${ }^{12}$ Fibre rotation also impacts the behaviour of individual particles through its effects on drag. ${ }^{2}$ In addition, for two particles in close proximity, collision or avoidance is dependent on rotation and orientation. ${ }^{13}$

The Stokesian dynamics method (SDM), based on the modified Jeffery's equation coupled to direct numerical simulation (DNS), has been used to study the dispersion, orientation, and deposition behaviours of axisymmetric ellipsoids in fully developed turbulent channel flows over a range of shear Reynolds numbers $\left(R e_{\tau}=u_{\tau} h / v=150-590\right)$, fibre aspect ratios $(\lambda=1-50)$, fibre non-dimensional relaxation times $\left(\tau_{p}^{+}=1-100\right)$, and fibre density ratios $\left(\rho_{p} / \rho \sim 7-3500\right){ }^{8,14-18}$ Here, $u_{\tau}$ is the shear (friction) velocity, $h$ is the channel half-height, $v$ is the fluid kinematic viscosity, $\rho$ and $\rho_{p}$ are the fluid and particle densities, respectively, and $\tau_{p}^{+}$is the ratio of the particle relaxation time $\left(\tau_{p}\right)$ to the fluid viscous time $\left(\tau_{f}=v / u_{\tau}^{2}\right)$. All these authors adopted the pointwise Lagrangian particle tracking (LPT) approach based on the assumption that the ellipsoids were smaller than the Kolmogorov length scale and that the flow around an ellipsoid is a creeping flow in the moving co-ordinates aligned with the ellipsoid's centre of mass. These studies considered dilute fibre suspensions, without taking into account the effect of fibre feedback to the flow, fibre-fibre interactions, and the effect of gravity. The authors also adopted idealised fibre-wall interactions, such that fibres were assumed to keep their streamwise and spanwise linear momenta, and the three components of angular momenta, when having elastic collisions with a wall. It has been reported ${ }^{15}$ that the transport properties of these ellipsoidal particles remain close to those of spherical particles. However, certain interesting concentration and orientation trends were observed which were in agreement with experimental observations. ${ }^{19-24}$ Similar to spheres, therefore, prolate spheroids were found to accumulate in the viscous sub-layer and preferentially concentrate in regions of low-speed streaks, with a strong dependence on their inertia $\left(\tau_{p}^{+}\right)$and a minor dependence on their aspect ratio. Being non-isotropic, ellipsoidal particles also tend to align with the mean flow direction, particularly very near the wall where their lateral tilting is suppressed. In the core region of the channel, the fibre orientation was found to be isotropic. Mortensen et al. ${ }^{15,16}$ and Zhao et al. ${ }^{17,18}$ also observed that the particle slip velocity $(\Delta \mathbf{u}=\mathbf{u}-\mathbf{v})$ is largely dependent on the particle's distance from the wall (a measure of the fluid velocity gradient), and that lighter particles show a positive slip velocity whilst heavier particles exhibit a negative slip velocity in the viscous sub-layer but a positive value in the buffer layer.

In spite of the considerable knowledge gained on fibre suspensions in turbulence from the application of Jeffery's equation ${ }^{25-27}$ for creeping flow and Stokes flow conditions, there are few 
studies on the effect of finite-sized particles on the physics of fibre suspensions undertaken using LPT. Simulations carried out using Stokesian models ${ }^{25,27}$ are only valid for particles with Reynolds numbers $\operatorname{Re}_{P} \leq 1$ and are not relevant for many engineering flows where the particles may be large with finite slip velocities and Reynolds numbers $R e_{P} \gg 1 .{ }^{28-31}$ Zastawny et al. ${ }^{30}$ recently applied fully resolved simulations using an immersed boundary method (IBM) and showed that the behaviour of spheroids at large slip velocities is quite different from that obtained using Stokesian models. ${ }^{25,27}$ Several recent papers have also reported results obtained using the lattice Boltzmann method (LBM), with fully resolved simulations of finite, neutrally buoyant spheroidal particles in shear flows, ${ }^{32-35}$ Couette flows, ${ }^{36,37}$ and turbulent channel flow. ${ }^{38}$ Depending on the particle shape and the inertia of the fluid and the particle, different particle motions were reported, for example, tumbling, wagging, kayaking, log-rolling, and even chaotic motions. Some of these modes of rotation have also been observed using large eddy simulation (LES) and LPT of finite-sized spheroids in turbulent channel flow. ${ }^{39}$

Currently, fully resolved simulations based on IBM and LBM are computationally expensive approaches which are not designed to handle suspensions of millions of small finite sized particles, as often met in engineering systems, and as required to obtain reliable particle statistics in a turbulent flow environment. To overcome the noted shortfalls of SDM, IBM, and LBM, and to exploit the enormous amount of information available from the use of LES and LPT, which are becoming increasingly used within industry, it is useful and necessary to employ LES-LPT to simulate spheroidal particle motion in turbulent flows. To address the challenge of specifying the particle drag coefficient, $C_{D}$, for particles of finite size, $d_{e v}$, and Reynolds number, $R e_{p}$, we present a modified Yin et al. ${ }^{40}$ approach to simulate the motion of spheroids beyond the Stokes regime in a Lagrangian framework. We propose the use of available empirical drag correlations expressed in terms of the equivalent diameter, $d_{e v}$, as a geometric parameter, and the aspect ratio, $\lambda$, and regular sphericity, $\Phi_{s}$, as shape factors in the empirical drag coefficient correlations to account for the departure in shape from a sphere, and use of the projected area, $A_{D}$, to account for the dependence on the particle dynamic orientation. The particle dynamic projected area is expressed by a functional relationship between the dynamic incidence angle, $\alpha$, and the particle's dimensions such as the particle semi-minor axis, $a$, and aspect ratio, $\lambda$. The particle incidence angle then links the fluid velocity gradient and the particle orientation. This method has been applied to model the motion of non-spherical particles, including cylinders in laminar flow, ${ }^{40,41}$ and spheres, needles, and disks in turbulent flow. ${ }^{39,42}$ Note that this method is different from common practice, e.g., ${ }^{43,44}$ where the inclusion of an equivalent diameter alone as the geometric parameter and optionally a shape factor, e.g., the sphericity, in the empirical drag coefficient is used to account for the departure in shape from a sphere, but the orientation dependency is ignored. This approach obviously oversimplifies the problem because a comprehensive account of the behaviour of non-spherical particles in turbulent flow requires that the drag force is treated with respect to the particle size, shape and orientation.

The present work concerns an extensive study of the rotation and orientation of large numbers of non-spherical particles in a turbulent channel flow. The particles are prolate ellipsoids, with aspect ratios greater than unity, which are heavier than the fluid (density ratio 760). Through variation of $a$ and $c$ a wide parameter space is explored, with two non-dimensional groups $\left(\lambda, \tau_{p}^{+}\right)$being varied independently whilst the density ratio $\left(\Phi_{\rho}=\rho_{p} / \rho\right)$ is kept constant. Different particle sets which cover a broad range of Stokes number are tracked and large particle samples are employed in order to ensure adequate convergence of the results and accurate particle statistics, ensuring a meaningful quantitative description of the phenomena under investigation. Results are obtained by analysis using the probability density function (PDF) formalism to describe the statistical variability in the orientational and rotational dynamics of anisotropic particles in turbulence.

The paper is organised as follows. In Section II, we describe the governing equations adopted in this work. In Section III, we discuss the physical problem, the LES code used to compute the turbulent flow field, and the Lagrangian particle tracker used in the Eulerian-Lagrangian approach. In Section IV, relevant results obtained in our simulations are given and discussed. Finally, the main findings are summarised and conclusions are drawn in the Section V. 


\section{GOVERNING EQUATIONS}

\section{A. Large eddy simulation}

Large eddy simulation resolves the large-scale energetic turbulent motions and models the sub-grid scale (SGS) motions. The top-hat filtered governing equations of motion for an incompressible fluid are given for mass and momentum, respectively, as

$$
\begin{gathered}
\frac{\partial \bar{u}_{j}}{\partial x_{j}}=0 \\
\frac{\partial \bar{u}_{i}}{\partial t}+\bar{u}_{j} \frac{\partial \bar{u}_{i}}{\partial x_{j}}=-\frac{1}{\rho} \frac{\partial \bar{p}}{\partial x_{i}}+\frac{\partial}{\partial x_{j}} \bar{\sigma}_{i j}-\frac{\partial}{\partial x_{j}} \tau_{i j},
\end{gathered}
$$

where $\bar{\sigma}_{i j}=-2 v \bar{s}_{i j}$ represents the viscous stress, $\bar{s}_{i j}=\frac{1}{2}\left(\partial \bar{u}_{i} / \partial x_{j}+\partial \bar{u}_{j} / \partial x_{i}\right)$ is the filtered strainrate tensor, $v$ is the kinematic viscosity, $\tau_{i j}=\bar{u}_{i} u_{j}-\bar{u}_{i} \bar{u}_{j}$ is the SGS tensor which represents the effect of the SGS motions on the resolved motions, $t$ is time, $x_{j}$ is the spatial co-ordinate directions, $u_{j}$ is the velocity vector, $p$ is the pressure, and $\rho$ is the density. The SGS tensor is computed using the dynamic version of the Smagorinsky model proposed by Piomelli and Liu. ${ }^{45}$ The eddy viscosity model of Smagorinsky ${ }^{46}$ for the SGS tensor linearly relates the anisotropic part of the SGS tensor $\tau_{i j}^{a}$ to the filtered strain-rate tensor, $\bar{S}_{i j}$, via an eddy viscosity coefficient, $\mu_{s g s}=\rho v_{s g s}$, with a length scale $\Delta=\left(\Delta_{x} \Delta_{y} \Delta_{z}\right)^{1 / 3}$ proportional to a measure of the grid spacing, hence

$$
\tau_{i j}^{a}=-2 v_{s g s} \bar{S}_{i j}
$$

where

$$
\begin{aligned}
& v_{s g s}=\left(C_{s} \bar{\Delta}\right)^{2}\|\overline{\mathbf{S}}\|, \\
& \|\overline{\mathbf{S}}\|=\sqrt{2 \bar{S}_{i j} \bar{S}_{i j} .}
\end{aligned}
$$

The model contains an adjustable parameter, $C_{s}$, often called the Smagorinsky constant which must be specified. However, as many previous studies have demonstrated and, consistent with the ideas of Kolmogorov, results are found to be relatively insensitive to the value of $C_{s}$; hence, the approximate localised dynamic model of Piomelli and $\mathrm{Liu}^{45}$ has been incorporated to calibrate $C_{s}$ as ${ }^{39,47}$

$$
\begin{gathered}
{\left[\left(C_{S}(\mathbf{x}, t)\right)\right]^{2}=\frac{\left[\overline{\left(C_{s}^{*}\right)^{2} \alpha_{i j}}-L_{i j}^{a}\right] \overline{\bar{S}_{i j}}}{2 \Delta_{T}^{2}\|\overline{\overline{\mathbf{S}}}\|\left(\overline{\bar{S}_{i j}}-\frac{\delta_{i j}}{3} \overline{\bar{S}_{k k}}\right) \overline{\bar{S}_{i j}}},} \\
\text { where } \alpha_{i j}=2 \Delta^{2}\|\overline{\mathbf{S}}\|\left(\bar{S}_{i j}-\frac{\delta_{i j}}{3} \bar{S}_{k k}\right) \\
\text { and } L_{i j}=\overline{\bar{u}_{i} \bar{u}_{j}}-\overline{\bar{u}}_{i} \overline{\bar{u}}_{j}
\end{gathered}
$$

Here, the overbar $(\bar{\bullet})$ denotes the application of a test filter of width $\Delta_{T}$ and $C_{S}^{*}$ is an approximation to $C_{s}$.

\section{B. Particle equation of motion}

\section{Kinematics}

The three reference frames shown in Fig. 1(a) are needed to model the particle dynamics. $\mathbf{x}^{\prime}=\left[x^{\prime}, y^{\prime}, z^{\prime}\right]$ represents the particle frame with its origin at the particle centroid and its axis being the principal axis, $z^{\prime}$, as opposed to the global (fixed) frame, $\mathbf{x}=[x, y, z]$. A third reference frame is $\mathbf{x}^{\prime \prime}=\left[x^{\prime \prime}, y^{\prime \prime}, z^{\prime \prime}\right]$ which is the co-moving frame with its origin coinciding with the particle centroid, and its axes being parallel to the fixed frame of reference. This work is based on a spheroidal particle which is characterised by three semi-axes $a, b$, and $c$ representing the radii in the $x^{\prime}-, y^{\prime}-$, and $z^{\prime}$-directions, respectively. The aspect ratio, $\lambda=c / a$, distinguishes between the axisymmetric $(a=b \neq c)$ prolate $(\lambda>1)$ and oblate $(\lambda<1)$ shapes and isotropic spherical $(\lambda=1)$ spheroids. 
(a)

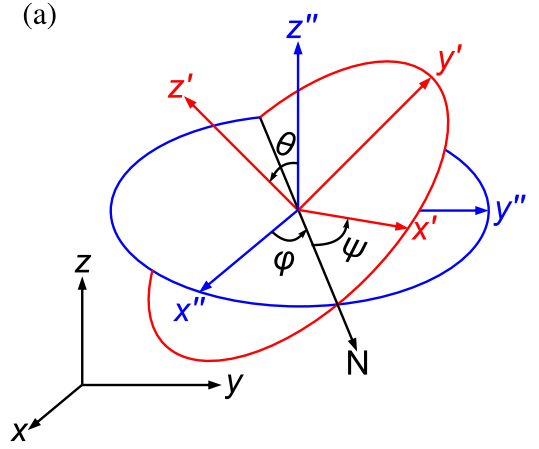

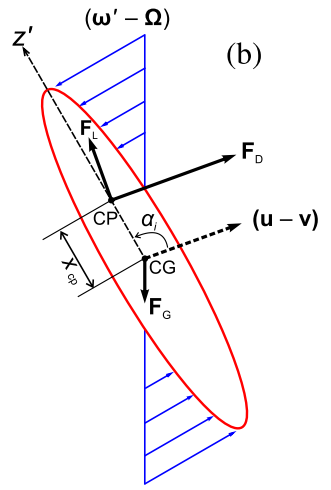

(c)

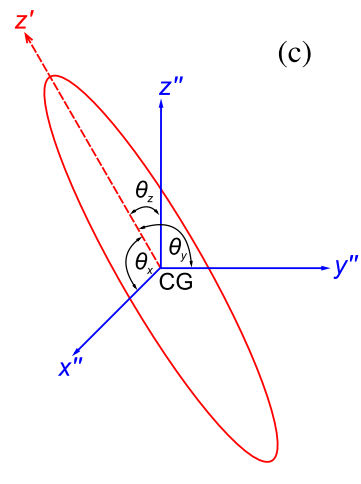

FIG. 1. (a) Relationship between the inertial $\mathbf{x}=[x, y, z]$, the particle $\mathbf{x}^{\prime}=\left[x^{\prime}, y^{\prime}, z^{\prime}\right]$, and the co-moving $\mathbf{x}^{\prime \prime}=\left[x^{\prime \prime}, y^{\prime \prime}, z^{\prime \prime}\right]$ co-ordinate systems and definition of the Euler angles $(\varphi, \theta, \psi) . \mathrm{N}=$ plane $\left[x^{\prime}, y^{\prime}\right] \cap$ plane $\left[x^{\prime \prime}, y^{\prime \prime}\right] . \theta$ is the angle between the $z^{\prime}$-axis and $z^{\prime \prime}, \varphi$ is the angle between the $\mathbf{x}^{\prime \prime}$-axis and $\mathrm{N}$, and $\psi$ is the angle between the $\mathbf{x}^{\prime}$-axis and $\mathrm{N}$. (b) Illustration of torque due to resistance on a rotating particle and location of the centres of gravity (CG) and pressure (CP), the incidence angle $\alpha_{i}$, and the forces acting on the particle. $x_{\mathrm{cp}}$ is the distance between CG and CP; the incidence angle $\alpha_{i}$ is the angle between the slip velocity $|\mathbf{u}-\mathbf{v}|$ and the particle major axis $\mathbf{z}^{\prime}$. (c) Angles $\theta_{x}, \theta_{y}$, and $\theta_{z}$ are, respectively, the angle between the particle principal axis $\mathbf{z}^{\prime}$ and its co-moving axes $x^{\prime \prime}, y^{\prime \prime}$, and $z^{\prime \prime}$.

The transformation between the co-moving frame and the particle frame is expressed as $\mathbf{x}^{\prime}=\mathbf{A} \mathbf{x}^{\prime \prime} . \mathbf{A}=\left[a_{i j}\right]$ is the time-dependent rotational matrix, and its elements, $a_{i j}$, can be expressed in terms of four quaternions $\mathbf{q}=\left[q_{0}, q_{1}, q_{2}, q_{3}\right]^{T}$ provided that the quaternions satisfy the constraint $q_{0}^{2}+q_{1}^{2}+q_{2}^{2}+q_{3}^{2}=1$. The orientational matrix is given as

$$
\mathbf{A}=\left[\begin{array}{lll}
1-2\left(q_{2}^{2}+q_{3}^{2}\right) & 2\left(q_{1} q_{2}+q_{0} q_{3}\right) & 2\left(q_{1} q_{3}-q_{0} q_{2}\right) \\
2\left(q_{1} q_{2}-q_{0} q_{3}\right) & 1-2\left(q_{1}^{2}+q_{3}^{2}\right) & 2\left(q_{2} q_{3}+q_{0} q_{1}\right) \\
2\left(q_{1} q_{3}+q_{0} q_{2}\right) & 2\left(q_{2} q_{3}-q_{0} q_{1}\right) & 1-2\left(q_{1}^{2}+q_{2}^{2}\right)
\end{array}\right],
$$

where the individual quaternions are obtained from the three Euler angles $(\varphi, \theta, \psi)$ as

$$
\begin{aligned}
q_{0} & =\cos \frac{\varphi+\psi}{2} \cos \frac{\theta}{2}, & q_{1} & =\cos \frac{\varphi-\psi}{2} \sin \frac{\theta}{2}, \\
q_{2} & =\sin \frac{\varphi-\psi}{2} \sin \frac{\theta}{2}, & q_{3} & =\cos \frac{\varphi+\psi}{2} \cos \frac{\theta}{2} .
\end{aligned}
$$

The time evolution of the quaternion is used to update the orientation of the particles and is computed as

$$
\frac{\mathrm{d} \mathbf{q}}{\mathrm{d} t}=\left(\begin{array}{c}
\dot{q}_{0} \\
\dot{q}_{1} \\
\dot{q}_{2} \\
\dot{q}_{3}
\end{array}\right)=\frac{1}{2}\left[\begin{array}{cccc}
q_{o} & -q_{1} & -q_{2} & -q_{3} \\
q_{1} & q_{0} & -q_{3} & q_{2} \\
q_{2} & q_{3} & q_{0} & -q_{1} \\
q_{3} & -q_{2} & q_{1} & q_{0}
\end{array}\right]\left[\begin{array}{c}
0 \\
\Omega_{x^{\prime}} \\
\Omega_{y^{\prime}} \\
\Omega_{z^{\prime}}
\end{array}\right],
$$

where $\Omega_{x^{\prime}}, \Omega_{y^{\prime}}$, and $\Omega_{z^{\prime}}$ are the components of the particle angular velocity in the particle frame. Fibre dispersion is treated in a Lagrangian framework following the method of Njobuenwu and Fairweather. ${ }^{39}$ The change in position of the $p$ th particle $\mathbf{x}_{\mathbf{p}}=\left[x_{p}, y_{p} z_{p}\right]$ is determined from the fibre stochastic velocity, $\mathbf{v}=\left[v_{x}, v_{y}, v_{z}\right]$ as

$$
\mathrm{d} \mathbf{x}_{\mathbf{p}}=\mathbf{v d} t
$$

\section{Dynamics of translational motion}

The acceleration of a stochastic particle in a turbulent flow field can be viewed as a random process, with position determined by a deterministic part evaluated in terms of filtered and known values and a stochastic component arising from the SGS turbulent motions of the fluid phase. In 
this study, only drag forces, $\mathbf{F}_{\mathbf{D}}$, taken from the Maxey and Riley ${ }^{48}$ formulation for the force per unit mass, and an additional force, the profile lift force $\mathbf{F}_{\mathbf{P L}}$, due to the orientation of the anisotropic particles ${ }^{40}$ are considered. A stochastic Markov model ${ }^{47,49}$ is used to represent the influence of the unresolved carrier fluid velocity fluctuations experienced by a stochastic particle $p$ over a time interval $\mathrm{d} t$ which is added to the deterministic contribution to give

$$
\mathrm{d} \mathbf{v}=\mathbf{A} \mathrm{d} t+\mathbf{B} \mathrm{d} \mathbf{w}_{\mathbf{t}}=\mathbf{F}_{\mathbf{D}} \mathrm{d} t+\mathbf{F}_{\mathbf{P L}} \mathrm{d} t+\left(C_{0} \frac{k_{s g s}}{\tau_{t}}\right)^{1 / 2} \mathrm{~d} \mathbf{w}_{\mathbf{t}} .
$$

The following assumptions are made to simply the simulation:

- The fluid velocity field is not affected by the motion of a particle, i.e., so-called one-way coupling is applied;

- the particles are large enough to neglect the influence of Brownian motion;

- the fluid-particle density ratio is small enough $\left(\rho / \rho_{p} \ll 1\right)$ to neglect buoyancy, added mass, pressure gradient, and Basset history forces. The shear lift force is also neglected;

- the effect of gravitational acceleration is neglected. As shown by Sardina et al., ${ }^{50}$ with large particle-fluid density ratios $\left(\rho_{p} / \rho \gg 1\right)$, the effect of gravity becomes negligible when the bulk velocity, $u_{b}$, is sufficiently large, considering the limit of large and small inertia. For large inertia, gravity effects are negligible when the Froude number $F r=u_{b} / \tau_{p} g \gg 1$. For our largest inertia, $\tau_{p}^{+}=100$ and $F r \sim 1.62 \gg 1$. For the smallest inertia, the gravity effect depends on the domain height and not on the relaxation time, $\tau_{p}$. In such cases, gravity effects are negligible when the Froude number $F r=u_{b} /(h g)^{0.5} \gg 1$. For our smallest inertia, $\tau_{p}^{+}=1$ and $F r \sim 4.06 \gg 1$;

- the flow considered is beyond Stokes conditions $\left(\operatorname{Re}_{P} \gg 1\right)$; hence, empirical correlations for the drag coefficient are employed.

The drag and profile lift forces in Eq. (10) acting on a single particle are expressed as ${ }^{40}$

$$
\begin{aligned}
\mathbf{F}_{\mathbf{D}} & =\frac{1}{2} \rho A_{D} C_{D}|\mathbf{u}-\mathbf{v}|(\mathbf{u}-\mathbf{v}), \\
\mathbf{F}_{\mathbf{P L}} & =\frac{1}{2} \rho A_{L} C_{L} \frac{\mathbf{z}^{\prime} .(\mathbf{u}-\mathbf{v})}{|\mathbf{u}-\mathbf{v}|}\left[\mathbf{z}^{\prime} \times(\mathbf{u}-\mathbf{v})\right] \times(\mathbf{u}-\mathbf{v}),
\end{aligned}
$$

where $\mathbf{u}=\left[u_{x}, u_{y}, u_{z}\right]$ and $\mathbf{v}=\left[v_{x}, v_{y}, v_{z}\right]$ are the fluid and particle velocity vectors at the particle position, $\mathbf{z}^{\prime}$ in Eq. (12) is the direction of the particle major axis, and $A_{D}$ and $A_{L}$ are the particle projected areas normal to the direction of the drag and profile lift forces, respectively. These projected areas are among those parameters that provide coupling between the translational and rotational motion and are expressed as a function of the particle incidence angle, $\alpha$, which is the angle between the particle slip velocity, $(\mathbf{u}-\mathbf{v})$, and the particle principal axis, $\mathbf{z}^{\prime}$ (see Fig. 1(b)), as $^{40}$

$$
\begin{aligned}
& A_{D}=\pi a^{2}\left[\cos ^{2} \alpha+(4 \lambda / \pi)^{2} \sin ^{2} \alpha\right]^{\frac{1}{2}}, \\
& A_{\mathrm{L}}=\pi a^{2}\left[\sin ^{2} \alpha+(4 \lambda / \pi)^{2} \cos ^{2} \alpha\right]^{\frac{1}{2}},
\end{aligned}
$$

where $A_{D}=\pi a^{2}$ for a spherical particle with aspect ratio $\lambda=1.0$. The drag coefficient, $C_{D}$ in Eq. (11), is obtained from a correlation for non-spherical particles available in the literature. The Ganser $^{51}$ drag coefficient correlation is adopted, which is based on the fact that every particle experiences a drag force according to Stokes' law, where drag is linearly related to the velocity, and Newton's law, where drag is proportional to the square of the velocity,

$$
\frac{C_{D}}{K_{2}}=\frac{24}{\operatorname{Re}_{p} K_{1} K_{2}}\left[1+0.118\left(\operatorname{Re}_{p} K_{1} K_{2}\right)^{0.6567}\right]+\frac{0.4305}{1+3305 /\left(\operatorname{Re}_{p} K_{1} K_{2}\right)},
$$

where $C_{\mathrm{D}}$ and $R e_{p}=|\mathbf{u}-\mathbf{v}| d_{e v} / v$ are based on the equal volume sphere diameter, $d_{e v}, K_{1}=$ $\left(\frac{1}{3} d_{n} / d_{e v}+\frac{2}{3} \Phi_{s}^{-0.5}\right)$ and $K_{2}=10^{1.8148\left(-\log \Phi_{s}\right)^{0.5743}}$ are the Stokes and Newton shape factors that model the particle sphericity and orientation, respectively, $d_{n}=\left(4 A_{D} / \pi\right)^{0.5}$ is the equal-projected 
area circle diameter, and $\Phi_{s}=s / S$ is the particle sphericity, defined as the ratio of the surface area of a sphere having the same volume as the particle, $s$, to the actual surface area of the non-spherical particle, $S$. The lift coefficient $C_{L}$ in Eq. (12) is determined such that the ratio of lift to drag forces satisfies the relationship, ${ }^{40}$

$$
\frac{\left|C_{L}\right|}{\left|C_{D}\right|}=\left|\sin ^{2} \alpha \cos ^{2} \alpha\right|
$$

Note that the validity of the equation for the translational motion in the inertial frame has been reported elsewhere. ${ }^{39,42}$

The last term in Eq. (10) represents the acceleration of the $p$ th stochastic particle via the diffusion process, taken to be isotropic, in which $k_{\mathrm{sgs}}$ is used to characterise the velocity fluctuations, $C_{0}$ is a model constant taken as unity, and $\mathrm{dW}_{\mathbf{t}}$ represents the increment of the Wiener process. ${ }^{47}$ During the simulation, the latter term is represented by $\xi_{i} \times \sqrt{\Delta t}$, where $\xi_{i}$ is a random variable sampled from a normal distribution with zero mean and a variance of unity, and which is independent for each time step and for each velocity component. $\tau_{t}$ in Eq. (10) is a sub-grid time scale which determines the rate of interaction between the particle and the turbulence dynamics, defined as $^{47,49}$

$$
\tau_{t}=\tau_{e v}\left(\frac{\tau_{e v} k_{s g s}^{1 / 2}}{\Delta}\right)^{0.6},
$$

which has been shown ${ }^{47,49}$ to reproduce the high values of kurtosis and PDFs observed experimentally. The model has also been shown to be capable of representing accurately particle dispersion in a droplet-laden turbulent mixing layer ${ }^{47} \Delta$ is the filter width, again given by $\Delta=\left(\Delta_{x} \Delta_{y} \Delta_{z}\right)^{1 / 3}$. The SGS kinetic energy, $k_{\mathrm{sgg}}$, is obtained using equilibrium arguments from $k_{\mathrm{sgs}}=2 \Delta C_{s}^{2 / 3} \bar{S}_{i j} \bar{S}_{i j}$.

The rotational motion of an ellipsoid about its principal axes is given by Euler's equation as

$$
\begin{aligned}
\frac{\mathrm{d} \Omega_{x^{\prime}}}{\mathrm{d} t} & =\frac{T_{x^{\prime}}}{I_{x^{\prime} x^{\prime}}}+\left(\frac{I_{y^{\prime} y^{\prime}}-I_{z^{\prime} z^{\prime}}}{I_{x^{\prime} x^{\prime}}}\right) \Omega_{y^{\prime}} \Omega_{z^{\prime}}, \\
\frac{\mathrm{d} \Omega_{y^{\prime}}}{\mathrm{d} t} & =\frac{T_{y^{\prime}}}{I_{y^{\prime} y^{\prime}}}+\left(\frac{I_{z^{\prime} z^{\prime}}-I_{x^{\prime} x^{\prime}}}{I_{y^{\prime} y^{\prime}}}\right) \Omega_{z^{\prime}} \Omega_{x^{\prime}}, \\
\frac{\mathrm{d} \Omega_{z^{\prime}}}{\mathrm{d} t} & =\frac{T_{z^{\prime}}}{I_{z^{\prime} z^{\prime}}}+\left(\frac{I_{x^{\prime} x^{\prime}}-I_{y^{\prime} y^{\prime}}}{I_{z^{\prime} z^{\prime}}}\right) \Omega_{x^{\prime}} \Omega_{y^{\prime}} .
\end{aligned}
$$

The principal moments of inertia of a spheroid are $I_{x^{\prime} x^{\prime}}=I_{y^{\prime} y^{\prime}}=\frac{1}{5} m_{p} a^{2}\left(1+\lambda^{2}\right)$ and $I_{z^{\prime} z^{\prime}}=\frac{2}{5} m_{p} a^{2}$. The net torque acting on the particle is $\mathbf{T}=\mathbf{T}_{\mathrm{H}}+\mathbf{T}_{\mathrm{R}}$. Here, $\mathbf{T}_{\mathrm{H}}$ is the torque due to the noncoincidence of the centres of gravity (CG) and pressure (CP), which takes care of the hydrodynamic force effect as illustrated in Fig. 1(b). Aerodynamic forces act at CP which is located a distance, $x_{c p}$, from CG. Using the particle major axis, $\mathbf{z}^{\prime}$, and the transformation matrix, $\mathbf{A}, \mathbf{T}_{\mathbf{H}}$ is given in the particle frame of reference as ${ }^{40}$

$$
\mathbf{T}_{\mathbf{H}}=\mathbf{A}\left[\left(x_{\mathrm{cp}} \mathbf{z}^{\prime}\right) \times\left(\mathbf{F}_{\mathbf{D}}+\mathbf{F}_{\mathbf{L}}\right)\right]
$$

where $\mathbf{T}_{\mathbf{H}}$ will change its sign, such that $\mathbf{T}_{\mathbf{H}}=-\mathbf{T}_{\mathbf{H}}$, when $\cos \alpha>0$, and

$$
x_{\mathrm{cp}}=0.25 c\left(1-e^{3(1-\lambda)}\right)\left|\cos ^{3} \alpha\right|,
$$

which is based on a semi-empirical expression proposed in the work of Yin et al. ${ }^{40}$ The components of torque due to the resistance on a rotating body, $\mathbf{T}_{\mathbf{R}}=\left[T_{x^{\prime}}, T_{y^{\prime}}, T_{z^{\prime}}\right]$, acting on the ellipsoidal particle are given as ${ }^{52}$

$$
\begin{aligned}
& T_{x^{\prime}}=\frac{16 \pi \mu a^{3} \lambda}{3\left(\beta_{0}+\lambda^{2} \gamma_{0}\right)}\left[\left(1-\lambda^{2}\right) d_{z^{\prime} y^{\prime}}+\left(1+\lambda^{2}\right)\left(\omega_{z^{\prime} y^{\prime}}-\Omega_{x^{\prime}}\right)\right], \\
& T_{y^{\prime}}=\frac{16 \pi \mu a^{3} \lambda}{3\left(\alpha_{0}+\lambda^{2} \gamma_{0}\right)}\left[\left(\lambda^{2}-1\right) d_{x^{\prime} z^{\prime}}+\left(1+\lambda^{2}\right)\left(\omega_{x^{\prime} z^{\prime}}-\Omega_{y^{\prime}}\right)\right], \\
& T_{z^{\prime}}=\frac{32 \pi \mu a^{3} \lambda}{3\left(\alpha_{0}+\beta_{0}\right)}\left(\omega_{y^{\prime} x^{\prime}}-\Omega_{z^{\prime}}\right),
\end{aligned}
$$


where $d_{z^{\prime} y^{\prime}}$ and $d_{x^{\prime} z^{\prime}}$ are elements of the deformation rate tensor and $\omega_{z^{\prime} y^{\prime}}, \omega_{x^{\prime} z^{\prime}}$, and $\omega_{y^{\prime} x^{\prime}}$ are elements of the spin tensor, defined as

$$
\begin{aligned}
& d_{z^{\prime} y^{\prime}}=\frac{1}{2}\left(\frac{\partial u_{z^{\prime}}}{\partial y^{\prime}}+\frac{\partial u_{y^{\prime}}}{\partial z^{\prime}}\right), \quad d_{x^{\prime} z^{\prime}}=\frac{1}{2}\left(\frac{\partial u_{x^{\prime}}}{\partial z^{\prime}}+\frac{\partial u_{z^{\prime}}}{\partial x^{\prime}}\right), \\
& \omega_{z^{\prime} y^{\prime}}=\frac{1}{2}\left(\frac{\partial u_{z^{\prime}}}{\partial y^{\prime}}-\frac{\partial u_{y^{\prime}}}{\partial z^{\prime}}\right), \quad \omega_{x^{\prime} z^{\prime}}=\frac{1}{2}\left(\frac{\partial u_{x^{\prime}}}{\partial z^{\prime}}-\frac{\partial u_{z^{\prime}}}{\partial x^{\prime}}\right) \omega_{y^{\prime} x^{\prime}}=\frac{1}{2}\left(\frac{\partial u_{x^{\prime}}}{\partial y^{\prime}}-\frac{\partial u_{y^{\prime}}}{\partial x^{\prime}}\right) .
\end{aligned}
$$

In Eqs. (24) and (25), the flow velocity gradients in the particle frame $G_{i^{\prime} j^{\prime}}$ can be obtained by transforming the velocity gradients from the fixed frame, $G_{i j}=\partial u_{i} / \partial x_{j}$, according to

$$
G_{i^{\prime} j^{\prime}}=\mathbf{A} G_{i j} \mathbf{A}^{-T}
$$

The non-dimensional coefficients $\alpha_{0}, \beta_{0}$, and $\gamma_{0}$ for a prolate ellipsoid $(\lambda>1)$ are defined as

$$
\begin{aligned}
& \alpha_{0}=\beta_{0}=\frac{\lambda^{2}}{\lambda^{2}-1}+\frac{\lambda}{2\left(\lambda^{2}-1\right)^{3 / 2}} \ln \left(\frac{\lambda-\sqrt{\lambda^{2}-1}}{\lambda+\sqrt{\lambda^{2}-1}}\right), \\
& \gamma_{0}=\frac{2}{\lambda^{2}-1}+\frac{\lambda}{\left(\lambda^{2}-1\right)^{3 / 2}} \ln \left(\frac{\lambda-\sqrt{\lambda^{2}-1}}{\lambda+\sqrt{\lambda^{2}-1}}\right) .
\end{aligned}
$$

\section{Ellipsoidal particle-wall collision models}

A spherical particle $(\lambda=1)$ is assumed to have contacted the wall when the distance from its centroid to the wall surface is smaller than its radius $\left(x_{p} \leq d_{p} / 2\right)$. An ellipsoidal particle is assumed to have touched the wall when the distance of its centroid to the wall varies between the semi-minor axis, $a$, and the semi-major axis, $c$, depending on the particle orientation. However, the probability of an ellipsoidal particle colliding with the wall depends on the distance of its centroid from the wall surface and its orientation relative to the wall. An ellipsoidal particle is considered to collide with the wall as soon as its tip touches the wall. Once a collision is detected, the particle is reflected elastically back into the flow. In such cases, the particle linear momentum in the wall-normal direction is changed in sign, whereas the linear momentum in the two homogenous directions and the angular momentum are unchanged. ${ }^{39} \mathrm{Fan}$ and $\mathrm{Ahmadi}^{52}$ developed a general approach to find the touch point of an ellipsoid on an arbitrary given wall. However, their method is cumbersome to implement. The method adopted here, although analogous to that of Fan and Ahmadi ${ }^{52}$ and Kim and Klapperich, ${ }^{53}$ is simpler. If the unit vector $[0,0,1]$ is attached to the ellipsoid major principle axis ( $\mathbf{z}^{\prime}$-axis) in the particle frame, the projection of this unit vector onto the local co-moving frame is given by

$$
\mathbf{r}_{\text {rot }}=\mathbf{A}^{\mathbf{T}}\left[\begin{array}{lll}
0, & 0 & 1
\end{array}\right]^{\mathrm{T}},
$$

and upon normalisation, the components of the orientation vector are given by

$$
\left[\begin{array}{l}
x_{r o t} \\
y_{\text {rot }} \\
z_{\text {rot }}
\end{array}\right]=\frac{2}{\left|\mathbf{r}_{\text {rot }}\right|}\left[\begin{array}{l}
q_{1} q_{3}+q_{0} q_{2} \\
q_{2} q_{3}-q_{0} q_{1} \\
q_{0}^{2}+q_{3}^{2}-\frac{1}{2}
\end{array}\right] .
$$

The ellipsoid length is defined as $L_{p}=a \lambda$, whereby the ellipsoid end points are located at

$$
\mathbf{x}_{\mathbf{B}}=\mathbf{x}_{\mathbf{p}} \pm \frac{1}{2} L_{p} \mathbf{x}_{\mathbf{r o t}},
$$

where $\mathbf{x}_{\mathbf{p}}$ is the position of centroid of the ellipsoid. An ellipsoidal particle is considered to collide with the wall as soon as its tip touches a wall. For the configuration considered, the two parallel walls are in the $y z$-plane; hence, the potential touch point of the ellipsoid with the wall $\left(x_{p}^{S}, x_{p}^{N}\right)$, 
with respect to the $\mathbf{x}^{\prime \prime}$ co-ordinate system, is given by expansion of Eq. (31) as

$$
\begin{aligned}
& x_{p}^{S}=x_{p}-\frac{1}{2} L_{p} x_{\text {rot }}, \\
& x_{p}^{N}=x_{p}+\frac{1}{2} L_{p} x_{\text {rot }} .
\end{aligned}
$$

\section{NUMERICAL METHODOLOGY}

The governing equations of turbulent flow between two parallel and horizontal walls, driven by a pressure gradient, were solved using the BOFFIN LES code. ${ }^{54}$ BOFFIN is a finite-volume code, with a co-located grid arrangement of the primary variables, which is based on a fully implicit low-Mach number formulation and is second-order accurate in both space and time. The carrier phase was air with density $\rho=1.3 \mathrm{~kg} \mathrm{~m}^{-3}$ and kinematic viscosity $v=1.57 \times 10^{-5} \mathrm{~m}^{2} \mathrm{~s}^{-1}$. The Reynolds number was $R e_{\tau}=h v_{\tau} / v=150$, based on the channel half-height, $h$, and the shear velocity, $u_{\tau}=\left(\tau_{w} / \rho\right)^{0.5}$, where $\tau_{w}$ is the mean wall shear stress. The channel's wall-normal, spanwise, and streamwise directions were the $x$-, $y$-, and $z$-axes, respectively. The computational domain was sized as $2 \pi \times 2 \pi h \times 4 \pi h$ which was discretised in physical space with $129 \times 128 \times 128$ grid points using a uniform mesh along the homogenous directions ( $y$-and $z$-axes). The mesh distribution in the wall-normal direction was specified using a non-uniform spacing according to a hyperbolic tangent series, ${ }^{55}$ given in Eq. (33), with a rational stretching factor, $\Phi_{c}$, such that $\Delta x_{\max } / \Delta x_{\min } \sim 100$

$$
\begin{gathered}
x_{i}=\frac{L_{x}}{2}\left[1+\Phi_{c} \tanh \left(K \eta_{i}\right)\right] \\
\text { with } K=\arg \tanh \left(\frac{1}{\Phi_{c}}\right) \\
\text { and }-1 \leq \eta_{i}=2 \frac{i-1}{N_{x}-1}-1 \leq+1 .
\end{gathered}
$$

With this computational domain, the spatial resolution in the spanwise and streamwise directions was, respectively, $\Delta y^{+} \sim 7.42$ and $\Delta z^{+} \sim 14.84$ in wall units, while in the wall-normal direction the minimum spacing (adjacent to the wall) was $\Delta x_{\min }^{+} \sim 0.07$ wall units, and the maximum spacing (at the centreline of the channel) was $\Delta x_{\max }^{+} \sim 7.08$ wall units. Here, that the superscript $\left(^{+}\right)$refers to a non-dimensional quantity scaled by the wall (viscous) variables, where $v_{\tau} / v, u_{\tau}$ and $v / u_{\tau}^{2}$ are the characteristic length, velocity, and time scales. To avoid the flow eventually becoming laminar, an initial simulation at high Reynolds number $\left(R e_{\tau} \sim 3000\right)$ was carried out using an equivalent bulk velocity, and the resulting solution was then used to initialise the flow at $R e_{\tau}=150$. Periodic boundary conditions were imposed in the streamwise and spanwise directions, while the no-slip condition was enforced at the walls.

The fibre simulation started after a fully developed fluid simulation was obtained. The fibre inertia is characterised using the non-dimensional particle relaxation time based on the viscous time scale $\left(\tau_{f}=u_{\tau}^{2} / v\right)$ and the equivalent volume of sphere diameter, $d_{e v}$, defined as $\tau_{e v}^{+}=\tau_{e v} / \tau_{f}=$ $\Phi_{\rho} d_{e v}^{+2} /(18 v)$ where $d_{e v}=2 a \lambda^{1 / 3}$. A total of 20 populations were considered, differing in the non-dimensional particle relaxation time, $\tau_{e v}^{+} \in[1,125]$, and particle aspect ratio, $\lambda \in[1,50]$. The 20 simulations evolved 200000 ellipsoids per population, with this number of particles ensuring results that were statistically convergent and independent of particle number, with some typical particle properties and related parameters listed in Table I. The lengths of the semi-axes were chosen such that the mass of particles with equal response times, $\tau_{e v}^{+}$, were equal. The density ratio of the fibre to the fluid was fixed at $\Phi_{\rho}=\rho_{p} / \rho \sim 760 .{ }^{56}$ We use the point-particle approximation on the assumption that the particles are smaller than the flow Kolmogorov length scale. ${ }^{8,57}$ The particle equations of translational and rotational motion were integrated using the fourth-order Runge-Kutta scheme. A sixth-order Lagrangian polynomial interpolation scheme ${ }^{8,56}$ was used to obtain the fluid velocity, SGS kinetic energy, and velocity gradients at a particle's position. The fibres' initial position was random, and their initial orientation was specified randomly using Euler angles ${ }^{58}$ such that 
TABLE I. Definition of the particle populations and parameters investigated. The number of particles per case was $N_{p}=200000$. The particle density ratio, $\Phi_{p}=\rho_{p} / \rho$, was fixed at approximately 760 in all cases. The non-dimensional relaxation time is based on the equivalent volume diameter, $\tau_{e q}^{+}$, whilst the particle aspect ratio, $\lambda$, of each population was fixed with the semi-minor axes, $a^{+}$and $c^{+}$, varied in each case together with the flow shear Reynolds number (where $\left.d_{e q}^{+}=f(a, \lambda), \lambda=c / a\right)$.

\begin{tabular}{|c|c|c|c|c|c|c|c|c|c|}
\hline Case & $\tau_{e q}^{+}$ & $\lambda$ & $2 c^{+}$ & $a^{+}$ & Case & $\tau_{e q}^{+}$ & $\lambda$ & $2 c^{+}$ & $d_{e q}^{+}$ \\
\hline St001Ar01 & 1 & 1.001 & 0.15 & 0.15 & St025Ar01 & 25 & 1.001 & 0.77 & 0.76 \\
\hline St001Ar03 & 1 & 3 & 0.32 & 0.15 & St025Ar03 & 25 & 3 & 1.59 & 0.76 \\
\hline St001Ar10 & 1 & 10 & 0.71 & 0.15 & St025Ar10 & 25 & 10 & 3.55 & 0.76 \\
\hline St001Ar30 & 1 & 30 & 1.48 & 0.15 & St025Ar30 & 25 & 30 & 7.38 & 0.76 \\
\hline St001Ar50 & 1 & 50 & 2.08 & 0.15 & St025Ar50 & 25 & 50 & 10.38 & 0.76 \\
\hline St005Ar01 & 5 & 1.001 & 0.34 & 0.34 & St125Ar01 & 125 & 1.001 & 1.53 & 1.53 \\
\hline St005Ar03 & 5 & 3 & 0.71 & 0.34 & St125Ar03 & 125 & 3 & 3.18 & 1.53 \\
\hline St005Ar10 & 5 & 10 & 1.59 & 0.34 & St125Ar10 & 125 & 10 & 7.10 & 1.53 \\
\hline St005Ar30 & 5 & 30 & 3.30 & 0.34 & St125Ar30 & 125 & 30 & 14.77 & 1.53 \\
\hline St005Ar50 & 5 & 50 & 4.64 & 0.34 & St125Ar50 & 125 & 50 & 20.76 & 1.53 \\
\hline
\end{tabular}

$\varphi \in[0,2 \pi] \theta \in[0, \pi / 2]$ and $\psi \in[0,2 \pi]$,

$$
\begin{aligned}
\varphi_{o} & =2 \pi \xi_{\phi}, \\
\theta_{0} & =\cos ^{-1}\left(1-\xi_{\theta}\right), \\
\psi_{0} & =2 \pi \xi_{\psi},
\end{aligned}
$$

where $\xi$ are random numbers with uniform distributions between 0 and 1 , and the subscripts $\varphi$, $\theta$, and $\psi$ represent the Euler number. The initial velocity and spin were set equal to those of the fluid at the particle position. Sufficient time was allowed (four eddy turnover times) for the particles to equilibrate with the flow before taking statistics. Periodic boundary conditions were applied in the homogeneous directions, while particle-wall collisions were fully elastic such that a spheroid kept its linear momentum in the two homogenous directions, as well as its angular momentum on touching the wall. ${ }^{57}$ The time step used for advancing the particle simulation was the same as for the fluid solution, i.e., $\Delta t^{+} \sim 6.192 \times 10^{-2}$ wall units.

\section{RESULTS AND DISCUSSION}

\section{A. Fluid phase}

First, the fluid phase LES results for the fully developed turbulent channel flow at low shear Reynolds number are analysed. Instantaneous contour plots of the streamwise velocity on the $x-y$ and $x-z$ planes are presented in Fig. 2, showing typical turbulent structures in the channel flow.

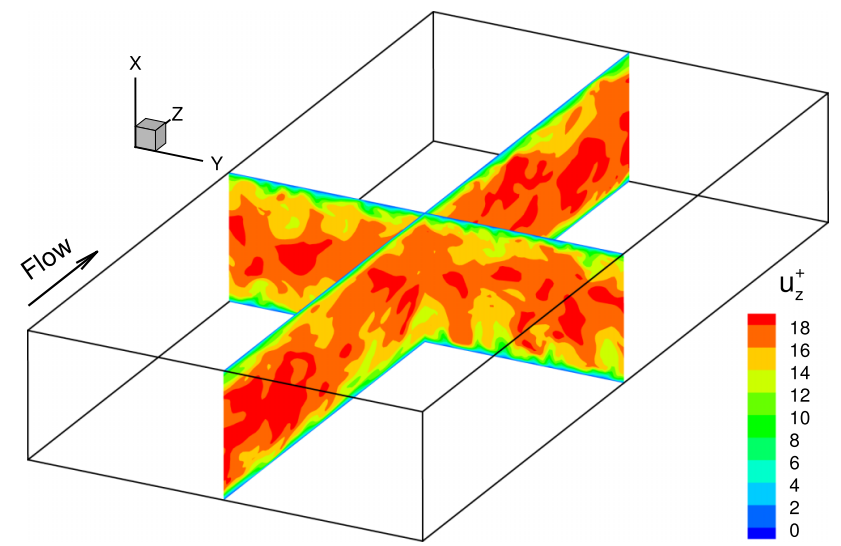

FIG. 2. Contours of instantaneous streamwise velocity normalised by wall variables, $u_{z}^{+}$, on $x-y$ and $x-z$ planes for present LES. 

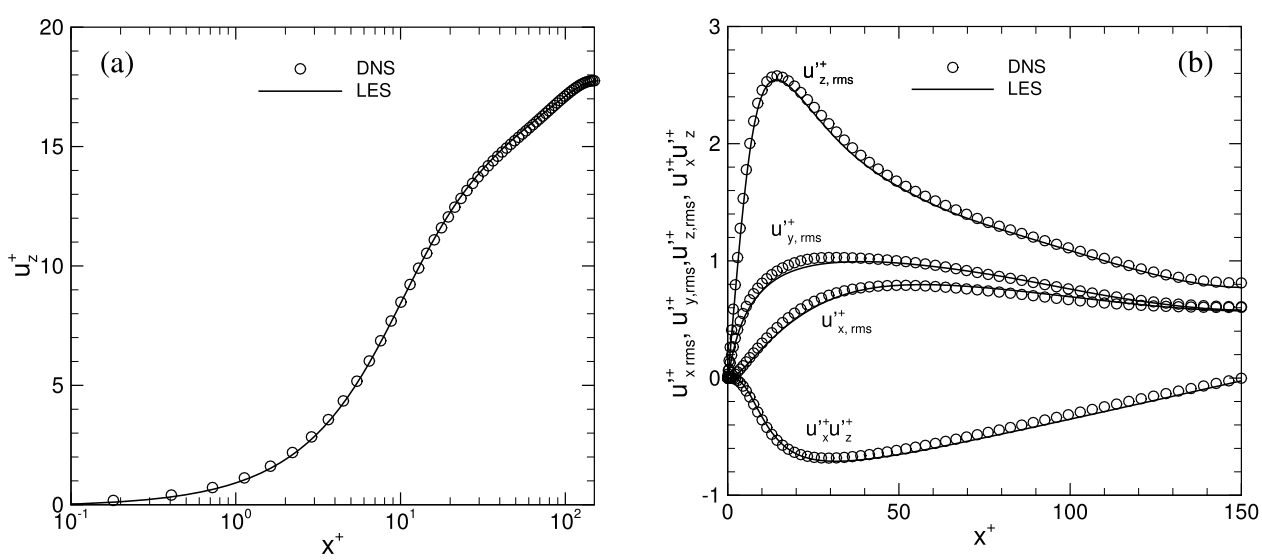

FIG. 3. Statistical moments of the turbulent channel flow: (a) mean streamwise velocity, $\left(u_{z}^{+}\right)$, and (b) wall-normal $\left(u_{x, \mathrm{rms}}^{\prime+}\right)$, spanwise $\left(u_{y, \mathrm{rms}}^{\prime+}\right)$, and streamwise $\left(u_{z, \mathrm{rms}}^{\prime+}\right)$ root mean square of velocity fluctuations and Reynolds shear stress $\left(u_{x}^{\prime+} u_{z}^{\prime+}\right)$.

Fig. 3(a) shows the profile of the mean streamwise velocity, $u_{z}^{+}=\left\langle u_{z}\right\rangle / u_{\tau}$, in the wall-normal direction, $x^{+}=x u_{\tau} / v$ (angular brackets $\langle\bullet\rangle$ indicate averaged in time and in the plane of statistical homogeneity, i.e., in the $y$-and $z$-axial directions), obtained for the $R e_{\tau}=150$ flow. The corresponding profiles of the root mean square (rms) of the fluctuating velocity components in the wall-normal, $u_{x, \mathrm{rms}}^{\prime+}=\left\langle u_{x, \mathrm{rms}}^{\prime}\right\rangle / u_{\tau}$, the spanwise, $u_{y, \mathrm{rms}}^{++}=\left\langle u_{y, \mathrm{rms}}^{\prime}\right\rangle / u_{\tau}$, and the streamwise, $u_{z, \mathrm{rms}}^{\prime+}=$ $\left\langle u_{z, \text { rms }}^{\prime}\right\rangle / u_{\tau}$, directions, and of the shear stress $u_{x}^{\prime+} u_{z}^{\prime+}=\left\langle u_{x}^{\prime} u_{z}^{\prime}\right\rangle / u_{\tau}^{2}$, are plotted in Fig. 3(b). The LES results are compared with those obtained from the DNS database of Marchioli et al. ${ }^{56}$ at the same shear Reynolds number, represented in the figures as symbols (o). The comparison shows very good agreement, confirming that the use of a highly resolved LES and dynamic modelling of the SGS term gives reliable results.

\section{B. Particle phase}

The phenomena investigated in this paper include the particle orientation with respect to the fluid slip velocity, the vorticity, and the inertial axes. Through variation of $\tau_{e v}^{+}, \lambda$, and $x^{+}$a wide parameter space has been investigated, as shown in Table I. Selected orientation distributions are shown, focusing on the importance of the velocity gradient, and the effect of the fibre aspect ratio and the fibre inertia. The orientation of the fibres is defined in terms of the alignment of the fibres' principal (main) axis with various directions in the flow, in our case, the fibre slip velocity direction, the vorticity direction, and the inertial (fixed) direction. For a boundary-layer type flow (e.g., channel flow), the homogeneity in the spanwise and streamwise directions means that our interest lies in variations in the inhomogeneous direction, i.e., the wall-normal direction. Below, results are therefore considered in terms of the three boundary layer regions of the flow: the viscous sub-layer $\left(0 \leq x^{+}<5\right)$, the buffer layer $\left(5 \leq x^{+}<50\right)$, and the log-law region $\left(50 \leq x^{+} \leq R e_{\tau}\right)$ which consequently studies the effect of the velocity gradient on fibre behaviour. In the subsequent text, values of the direction cosine, $\left|\cos \theta_{i}\right| \sim 1$, denote a fibre exhibiting strong alignment with (i.e., parallel to) the $i$ th direction, while values of $\cos \theta_{i}=0$ indicate fibres exhibiting strong alignment across (i.e., perpendicular to) the $i$ th direction; with $\theta$ being the angle, the principal axis, $z^{\prime}$, subtends to the direction of interest. Hereinafter, when discussing the orientation of a fibre, or another ellipsoidal particle, its principal axis, $z^{\prime}$, is implied, except otherwise stated.

To quantitatively analyse the fibres' alignment trends, we examine the probability density function of the direction cosine $\cos \theta_{i}$. The PDF is computed using $f(\xi)=N_{\xi} / N$, where $\xi=\Phi_{i}$ and $\Phi_{i}$ is the $i$ th variable of interest. Here, $N_{\xi}$ is the number of fibres, with $\xi$ being in the bin $[\xi, \xi+\Delta \xi]$, and $\Delta \xi=\left(\xi_{\max }-\xi_{\min }\right) / N_{B}$, with $N$ the total number of samples used and $N_{B}$ the number of bins. Note that the PDF satisfies the normalisation condition $\sum_{j=1}^{N_{B}} f\left(\xi_{j}\right)=1.0$. 


\section{Instantaneous fibre concentration}

An overall impression of the simulated fibre distribution, concentration, and alignment can been seen for selected wall-normal locations and fibre and fluid properties in Fig. 4. This figure presents the instantaneous streamwise velocity fluctuation field, $u_{z}^{\prime+}$, together with snapshots of individual fibres to provide a general impression of how the fibres are preferentially dispersed and

(a)

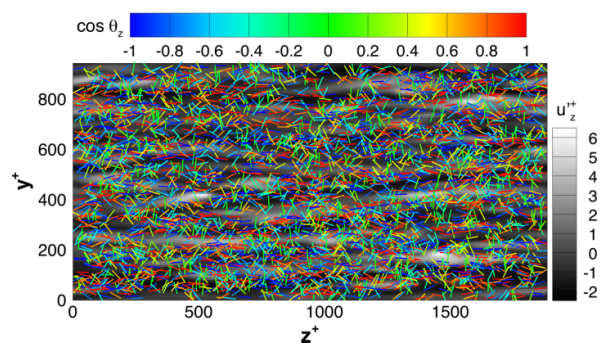

(b)

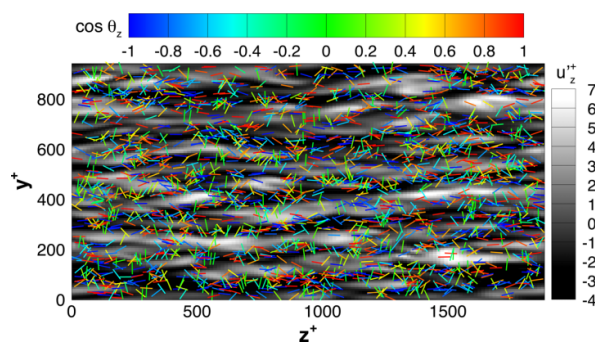

(c)

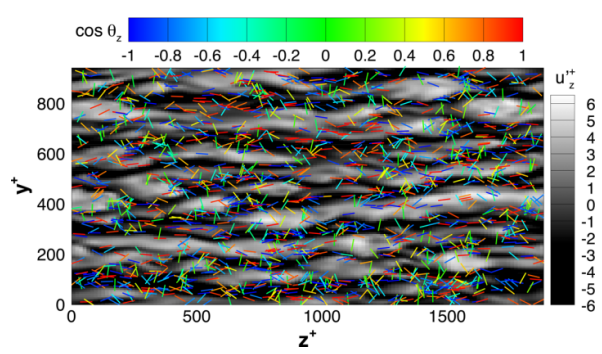

(d)

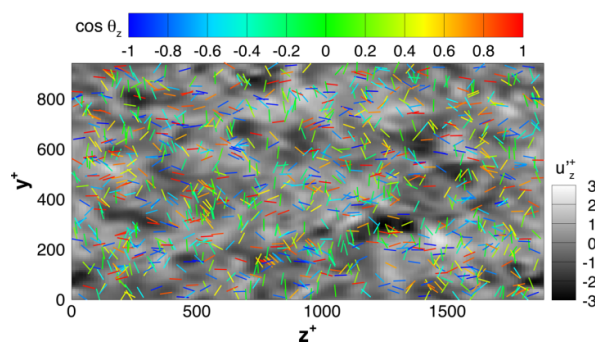

FIG. 4. Vector plot of particle orientation superimposed on a contour plot of the velocity field, showing instantaneous fibre distribution (mean flow direction from left to right). Panels represent snapshots taken at wall-normal distances of (a) $3.0 \leq x^{+} \leq 5$, (b) $7 \leq x^{+} \leq 9$, (c) $14 \leq x^{+} \leq 16$, and (d) $148 \leq x^{+} \leq 150$ for case St025Ar30. The mid-point of the fibre length (though not to scale) represents the positions of the fibre, while the arrow colours indicate alignment with the specified direction. 
orientated in the flow. The results focus on four specific regions in the flow. The near-wall plane $\left(3 \leq x^{+} \leq 5\right)$ in the viscous sub-layer region was selected as the velocity gradient here is high and it is at the point where fibres are trapped before deposition on the wall. ${ }^{59}$ The second plane is in the buffer region $\left(7 \leq x^{+} \leq 9\right)$, chosen as it is at a distance from the wall at which the magnitudes of the mean slip velocity and the slip velocity fluctuations reach a maximum for spherical particles. ${ }^{17,18}$ The next plane considered is also in the buffer region $\left(14 \leq x^{+} \leq 16\right)$ at the location where the magnitude of the turbulent velocity fluctuations reaches a maximum value, with the turbulence in this region characterised by strong ejection and sweep events. ${ }^{60}$ The fourth plane is located in the core of the flow and across the channel centerline $\left(148 \leq x^{+} \leq 150\right)$ where there is almost no mean shear and turbulence is nearly homogeneous and isotropic. The fluid velocity fields are sampled at the mid-points $x^{+}=4,8$, and 15 for Figs. 4(a)-4(c), and at the channel centreline $x^{+}=150$ for Fig. 4(d).

In Fig. 4 , the grey-scale contours represent the streamwise fluid velocity fluctuations, $u_{z}^{\prime+}=$ $u_{z}^{+}-\bar{u}_{z}^{+}$, where $u_{z}^{+}$and $\bar{u}_{z}^{+}$are the instantaneous and Eulerian spatially averaged streamwise components of the fluid velocity vector. The fibres' positions and orientations are superimposed on the fluid streamwise velocity fluctuation for the case of particles with inertia $\tau_{e v}^{+}=25$ and aspect ratio $\lambda=30$ from simulation case St025Ar30 (see Table I). Each fibre is represented by a solid straight line with its centre indicating the fibre position. The length of the line is arbitrarily fixed and is not scaled, although its orientation depicts the fibre alignment. The fibres are coloured by the fibre direction cosine, $\cos \theta_{z}$, where $\theta_{z}$ is the angle between the fibre's principal axis, $z^{\prime}$, and the direction of the flow (i.e., streamwise $z$ direction).

The streaky nature of the flow in the near-wall (viscous sub-layer and buffer) region in Figs. 4(a)-4(c) is clearly shown as elongated regions of low-speed fluid (dark areas) alternating with regions of fast-moving fluid (light areas). Although the fibres were initially uniformly present in the turbulent flow field, they tend to distribute themselves in the near-wall region, avoiding the high-speed regions and concentrating in the low-speed regions. This tendency for initially uniformly distributed inertial fibres to preferentially concentrate on low-speed streaks in the near-wall region is consistent with earlier observations for spherical $(\lambda=1)$ particles, ${ }^{61,62}$ as well as for both prolate $(\lambda>1)^{8,14-16,18}$ and oblate $(\lambda<1)^{57}$ particles. This effect is strongest for fibres in the viscous sub-layer region, Fig. 4(a), but diminishes as we move towards the centre-line, Fig. 4(d), where the particles are seen to be anisotropic in their distribution. This tendency is also evident in terms of the fibres' preferential concentration, with more fibres concentrated in the near-wall region in Fig. 4(a) due to the actions of ejection and sweep events than in Figs. 4(b)-4(d). As is evident from videos made from the simulation, the fibres are also observed to rotate faster in the near-wall region than in the core region of the channel.

The fibre alignment with the global (fixed) axes is significant since it can influence the rotation of the fibre and, as a consequence, the rheological and mechanical properties of suspensions populated with elongated fibres. It is known that the rotation (spinning or tumbling) rate of fibres in fluid flows is strongly dependent on the local velocity gradient of the flow and, consequently, it is expected that the fibre orientation correlates with the fluid rotation (which is equal to half the velocity gradient) at the position of the fibre. In Figs. 4(a)-4(c), the fibre colour coding reveals that most of the fibres in the near-wall region exhibit a strong preferential orientation with their principal axis, $z^{\prime}$, aligned with the streamwise direction, $z$-axis, such that $\left|\cos \theta_{z}\right| \sim 1$. In contrast, the fibres' orientation is isotropic, taking several different orientations, in the core region of the flow where the velocity fluctuations are almost isotropic, Fig. 4(d). Additionally, there is no orderly distribution of the fibre positions in this region of the flow. Again, the preferential orientation of the fibres observed in Fig. 4 is in qualitative agreement with previous findings. ${ }^{8,14-16,18}$ These observations demonstrate that the predictive approach described qualitatively captures fibre interactions with the flow, and these will be further substantiated to include the quantitative effects of fibre inertia and aspect ratio by means of statistical analysis in the remaining sections. 


\section{Alignment of fibres with slip velocity direction}

The orientation distribution of fibres can be considered relative to different directions defined by the flow. First, we consider alignment with the particle slip velocity direction, $\Delta \hat{\mathbf{u}}$, by monitoring the probability density function of $\cos \alpha=\mathbf{z}^{\prime} \cdot \Delta \hat{\mathbf{u}}$, where $\alpha$ is the so called incidence angle, i.e., the angle subtended by the particle principal axis, $\mathbf{z}^{\prime}$, to the slip velocity direction $\Delta \hat{\mathbf{u}}=\Delta \mathbf{u} /|\Delta \mathbf{u}|$, where the slip velocity is $\Delta \mathbf{u}=(\mathbf{u}-\mathbf{v})$. As any arbitrarily shaped particle can potentially take on any orientation, the incidence angle $\alpha$ illustrated in Fig. 1(b) must be determined for each time step and is used to consider the orientability and rotational motion of super ellipsoids due to the hydrodynamic forces and torques they experience. This allows insight into the relationship between the fibre orientation and the underlying flow field when varying the fibre shape and size.

First, to gain some basic insight into the dynamics of non-spherical particles, the probability density function of the direction cosine of the fibre incidence angle, $\operatorname{PDF}(\cos \alpha)$, is presented in Figs. 5 and 6. As noted earlier, sampling was performed at four wall-normal locations: at $\left(3 \leq x^{+} \leq 5\right)$ (within the viscous sub-layer), $\left(7 \leq x^{+} \leq 9\right)$ and $\left(14 \leq x^{+} \leq 16\right)$ (within the buffer region), and $148<x^{+} \leq 150$ (in the outer region of the flow). In Fig. 5, we keep the fibre inertia fixed at $\tau_{e v}^{+}=25$ and investigate the effect of the fibre aspect ratio, $\lambda=1-50$, while in Fig. 6 , we fix the aspect ratio at $\lambda=30$ and examine the influence of the fibre inertia, $\tau_{e v}^{+}=1-125$. In Fig. 5, the near spherical particles, $\lambda=1.001$, generally show a different behaviour to the elongated particles, $\lambda \gg 1$. The orientation of all fibres with respect to the slip velocity vector in the near-wall regions, Figs. 5(a) and 5(b), is also at variance with that obtained in the inertial regions in Figs. 5(c)
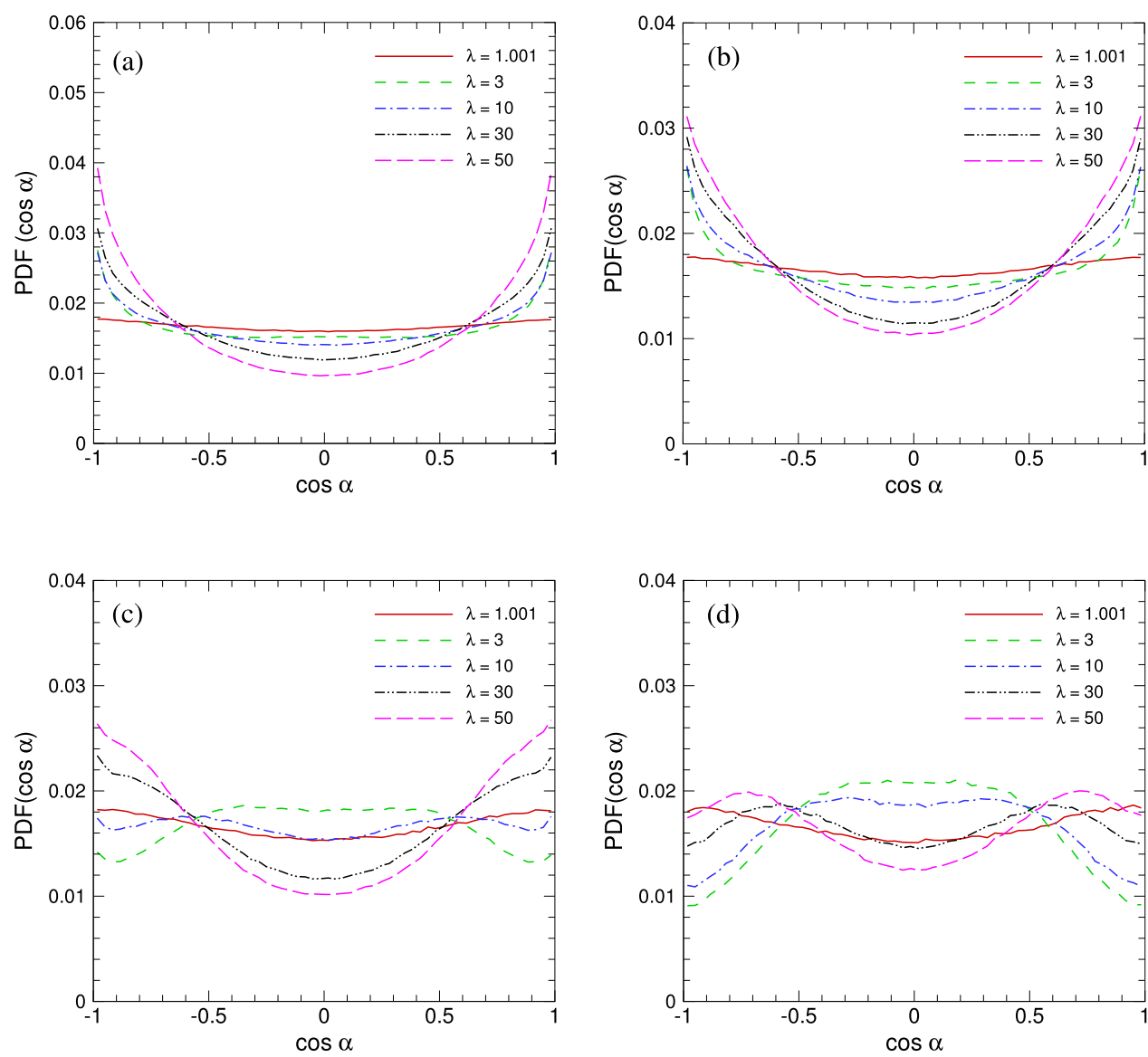

FIG. 5. Effect of channel location, $x^{+}$, and particle aspect ratio, $\lambda$, on the probability density function of the direction cosine, $\operatorname{PDF}(\cos \alpha)$, of the incidence angle, $\alpha$, between the fibre principal axis, $z^{\prime}$, and the flow slip velocity direction, $\Delta \mathbf{u} /|\Delta \mathbf{u}|$, at (a) $3 \leq x^{+} \leq 5$, (b) $7 \leq x^{+} \leq 9$, (c) $14 \leq x^{+} \leq 16$, and (d) $148 \leq x^{+} \leq 150$ with series representing $\lambda=(1.001,3,10,30,50)$ particles with a fixed particle inertia, $\tau_{e v}^{+}=25$. 

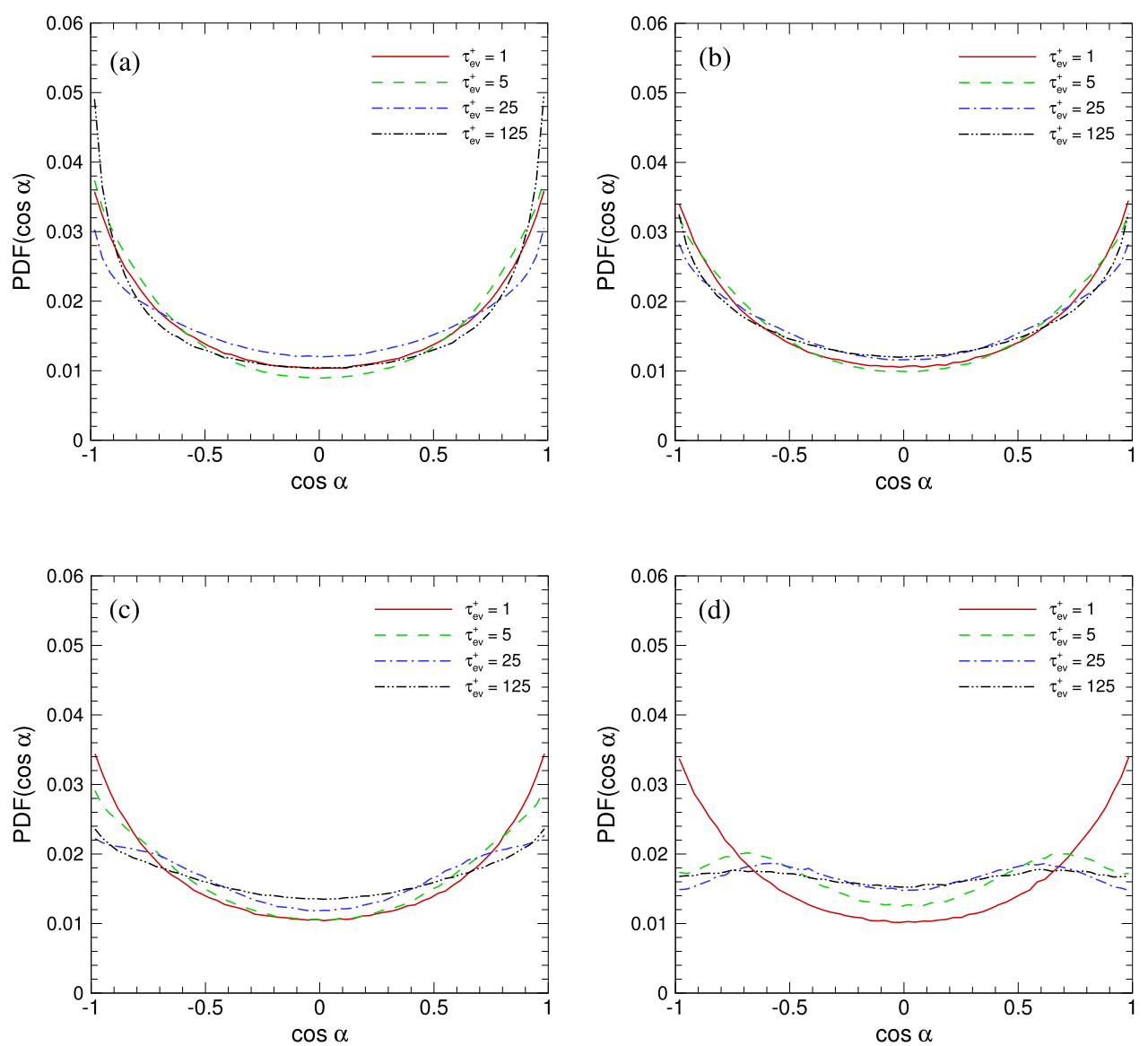

FIG. 6. Effect of channel location, $x^{+}$, and particle inertia, $\tau_{e v}^{+}$, on the probability density function of the direction cosine, $\operatorname{PDF}(\cos \alpha)$, of the incidence angle, $\alpha$, between the fibre principal axis, $z^{\prime}$, and the flow slip velocity direction, $\Delta \mathbf{u} /|\Delta \mathbf{u}|$, at (a) $3 \leq x^{+} \leq 5$, (b) $7 \leq x^{+} \leq 9$, (c) $14 \leq x^{+} \leq 16$, and (d) $148 \leq x^{+} \leq 150$ with series representing $\tau_{e v}^{+}=(1,5,25$, 125) particles with a fixed aspect ratio, $\lambda=30$.

and 5(d). In Figs. 5(a) and 5(b), the PDFs show peaks when the fibres' orientation is aligned with the fibre slip velocity direction. For a fixed inertia, $\tau_{e v}^{+}=25$, an increasing probability of parallel alignment with the slip velocity is seen at larger particle aspect ratios. The near-spherical particles show the least alignment with the slip velocity, although their orientation shows two small maxima at $|\cos \alpha|=1$, an indication that their orientation shows no real preferential alignment, as might be anticipated. Away from the near-wall region all the fibres exhibit a weaker alignment of their orientation with the slip velocity. In Fig. 5(c), larger aspect ratio $(\lambda=50$ and 30) fibres show peaks corresponding to parallel alignment, whereas the smaller aspect ratios only exhibit very small parallel alignment peaks, apart from the spherical particles. The smaller $\lambda=3$ and 10 fibres also exhibit slight maxima at $|\cos \alpha| \sim 0$.5. In Fig. 5(d), all the particles now show peaks away from $|\cos \alpha|=1$, with a gradual transition from spherical particles to $\lambda=3$ and 10 fibres which show preferential near-orthogonal alignment to larger aspect ratios $(\lambda=50$ and 30) which maintain peaks closer to $|\cos \alpha|=1$ For the particle shapes investigated, the alignment with the slip velocity therefore shows a strong dependence on the particle aspect ratio, with elongated fibres tending to show more preferential orientation to their slip velocity direction than their nearer-spherical counterparts.

The inertia (or size) of the fibre also affects its orientation with respect to its slip velocity vector. The distributions shown in Fig. 6 illustrate that all the fibres, irrespective of their inertia, tend to align with the slip velocity direction, with strong alignment in the near-wall regions, Figs. 6(a) and 6(b), but weaker alignment in the outer regions of the boundary layer Figs. 6(c) and 6(d). In the near-wall regions of the channel, the degree of alignment appears to be largely independent of the fibre inertia, save for the largest inertia case, $\tau_{e v}^{+}=125$, in Fig. 6(a) where lateral tilting and 
fibre-wall interactions were found to further enforce the strong alignment with the slip velocity vector. In the outer regions of the flow, the smallest fibres $\left(\tau_{\rho v}^{+}=1\right)$ show the strongest alignment with peaks at $\cos \alpha \sim|1|$, whereas a lesser degree of alignment between $z^{\prime}$ and $\Delta \mathbf{u} /|\Delta \mathbf{u}|$ is observed for the more inertial fibres $\left(\tau_{e v}^{+}>1\right)$, with the peaks shifting to $\cos \alpha \sim|0.6|$ in Fig. 6(d).

\section{Alignment of fibres with global axes}

To quantify the preferential alignment of anisotropic particles in turbulence, we now consider the alignment trends of the particle orientation with respect to the global (fixed) reference frame, $\mathbf{x}=[x, y, z]$. In doing this, attention is focussed on the angles, $\theta_{i=x, y, z}$, between the particle principal axis, $\mathbf{z}^{\prime}$, and the $\mathbf{x}$-directions of the inertial reference frame, as illustrated in Fig. 1(c). A qualitative illustration of how parameters such as the fibre aspect ratio influence the fibre orientation is given in the instantaneous plots of Fig. 7 for aspect ratios $\lambda=3$ (case St001Ar03) and $\lambda=50$ (case St001Ar50). The alignment trends with the fixed axes are quantified by evaluating the PDF of the direction cosine, $\cos \theta_{i=x, y, z}$, with the results given in Figs. 8-13 for the same four planes parallel to the wall as considered above, i.e., at $3 \leq x^{+} \leq 5,7<x^{+} \leq 9,14<x^{+} \leq 16$, and $148<x^{+} \leq 150$. The statistical analysis reported below complements the qualitative observations made in relation to the instantaneous plots of fibre alignment with the fixed axes given in Fig. 4.

Results are shown in Fig. 8 for the alignment of the fibre principal axis, $z^{\prime}$, with the flow direction, $\mathrm{z}$-axis, as a function the particle anisotropy parameter, $\lambda=1.001,3,10,30$, and 50, for a fixed fibre inertia parameter, $\tau_{e v}^{+}=25$. Similar results are shown in Fig. 9 for a fixed fibre aspect ratio, $\lambda=30$, and varying the fibre inertia, $\tau_{e v}^{+}=1,5,25$, and 125. In Figs. 8(a)-8(c) and 9(a)-9(c), the $\operatorname{PDF}\left(\cos \theta_{z}\right)$ has three extrema: a minimum at $\cos \theta_{z}=0$ and two maxima at $\cos \theta_{z}=-1$ and $\cos \theta_{z}=1$, demonstrating strong fibre alignment, or orientation anisotropy with symmetric alignment, with an equal probability of their orientation being with the positive and negative $z$-axis. The near-spherical $\left(\tau_{e v}^{+}=25, \lambda=1.001\right)$ particle is an exception, for the St025Ar01 case in Figs. 8(a)-8(c), where these particles mimic spherical particles and show no preferential alignment. This is also the case for all fibres sampled at the channel centre position $\left(148 \leq x^{+} \leq 150\right)$ in Figs. 8(d) and 9(d). The PDF $\left(\cos \theta_{z}\right)$ of the St025Ar01 case and of all fibres at the channel centre therefore shows a flat profile with a roughly constant value which only varies by approximately $2 \%$. Hence, as expected, the isotropy of the turbulence at the channel centre causes a randomising effect on fibre orientation at this location.

(a)

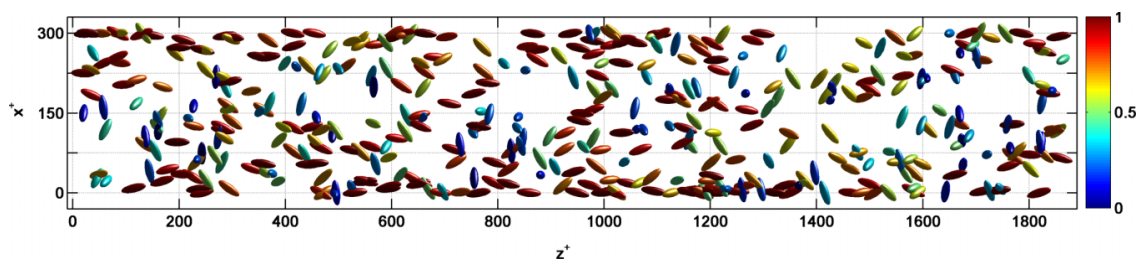

(b)

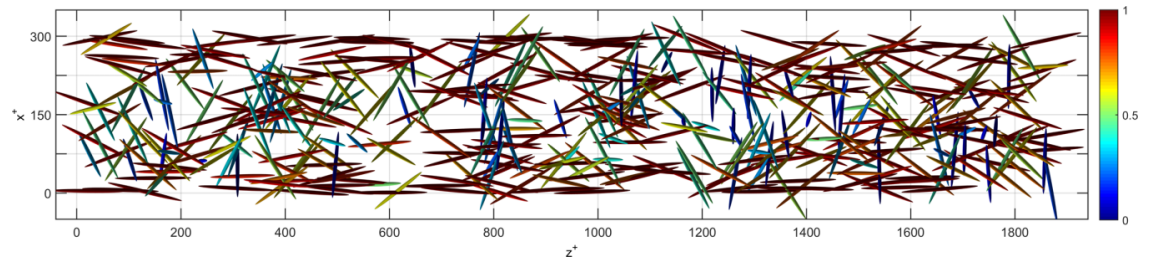

FIG. 7. Instantaneous distribution of fibre concentration and orientation showing effect of aspect ratio: (a) $\lambda=3$, case St001Ar03, and (b) $\lambda=50$, case St001Ar50, for a fixed inertia, $\tau_{e v}^{+}=1$. The fibres are projected onto the ( $\left.x, z\right)-$ plane, with the fibre shape indicating orientation and the centre of mass indicating position. Colour-coding is for the direction cosine of the angle $\theta_{z}$ between the particle major axis, $\mathbf{z}^{\prime}$, and the streamwise direction, $z$-axis. The fibres' size is scaled relative to the $(x, z)$-plane by a factor of 150 for clarity. 

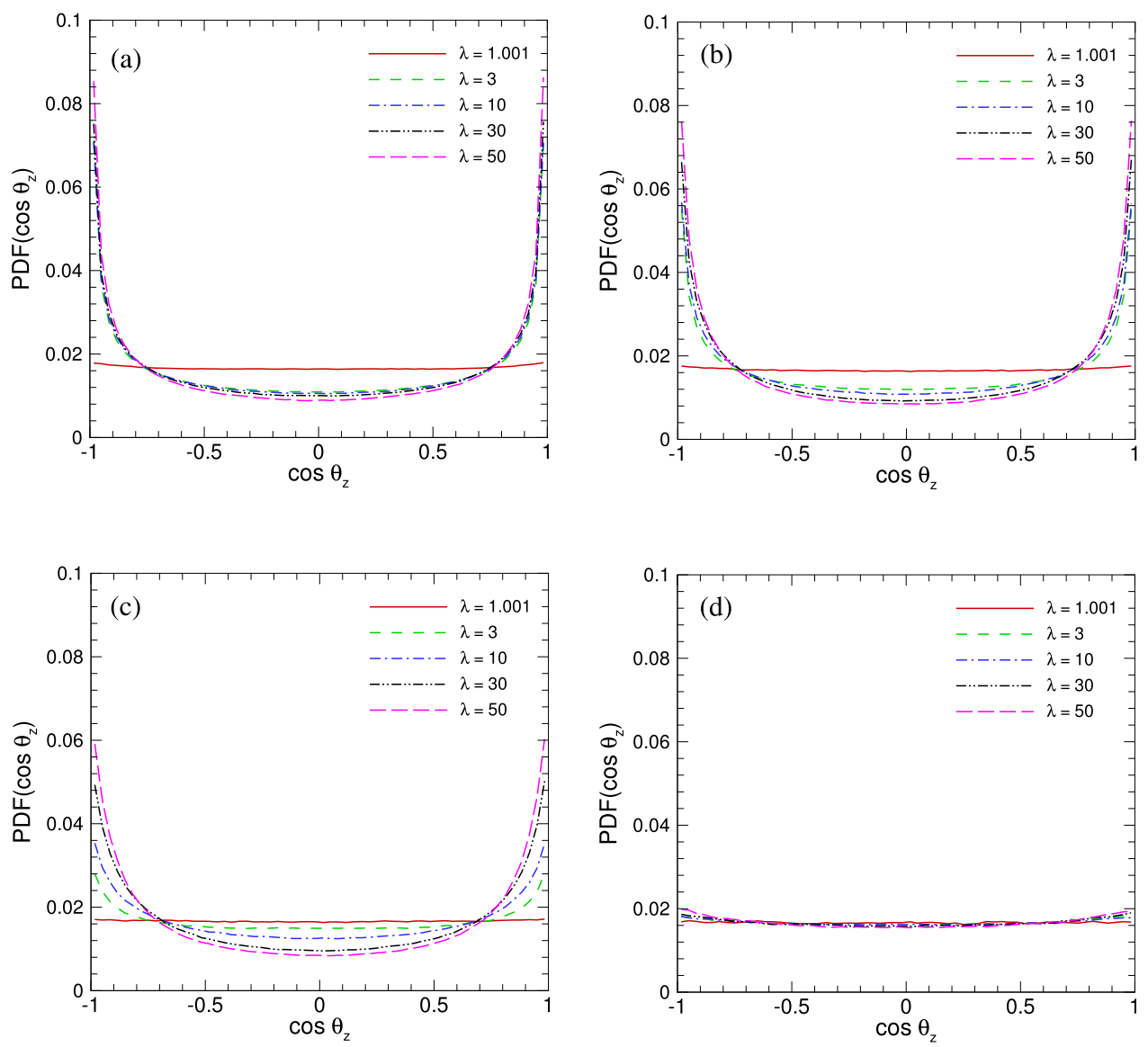

FIG. 8. Effect of channel location, $x^{+}$, and particle aspect ratio, $\lambda$, on the probability density function of the direction cosine, $\operatorname{PDF}\left(\cos \theta_{z}\right)$, of the angle $\theta_{z}$ between the fibre principal axis, $z^{\prime}$, and the global $z$-axis at (a) $3 \leq x^{+} \leq 5$, (b) $7 \leq x^{+} \leq 9$, (c) $14 \leq x^{+} \leq 16$, and (d) $148 \leq x^{+} \leq 150$ with series representing $\lambda=(1.001,3,10,30,50)$ particles with a fixed particle inertia, $\tau_{e v}^{+}=25$.

With respect to the fibre aspect ratio (elongation) in Figs. 8(a)-8(c), as noted, the fibres tend to align themselves with the flow direction as the $\operatorname{PDF}\left(\cos \theta_{z}\right)$ peaks at $\left|\cos \theta_{z}\right| \sim 1$. Hence, in these locations, namely the viscous sub-layer, Fig. 8(a), and the buffer layer, Figs. 8(b) and 8(c), the fibres exhibit more spinning (i.e., rotation about their principal $z^{\prime}-$ axis) than they do in the channel centre region where the fluid velocity gradient is low. Similar to the observation of Vincenzi, ${ }^{63}$ the degree of fibre alignment with, and rotation about, the $z^{\prime}$-axis is strongly dependent on the fibre elongation and the flow turbulence anisotropy. Considering the fibre orientation statistics sampled at each location, the degree of alignment of the fibre principal axis with the direction of flow decreases as the shape of the fibre approaches near-spherical with $\lambda=1.001$. Moving from Fig. 8(a) to Fig. 8(d), the degree of alignment of the principal axis, $z^{\prime}$, of the elongated fibres $(\lambda=3,10,30$, and 50) with the flow direction, z-axis, decreases as the degree of anisotropy of the flow decreases in moving towards the channel centre. In close proximity to the wall, the fibres exhibit the highest preferential alignment to the flow direction, with this due to the combined effect of a large velocity gradient at this location and the suppression of lateral tilting of the elongated particles. The peak values of the PDF for the most aligned fibre, i.e., the $\lambda=50$ case, reduce from approximately $8.5 \%$ in the viscous sub-layer $\left(3 \leq x^{+} \leq 5\right)$, Fig. $8(a)$, to about $7.6 \%$ at the first buffer layer position $\left(7 \leq x^{+} \leq 9\right)$, Fig. 8(b), to $6 \%$ at the second buffer layer position $\left(14 \leq x^{+} \leq 16\right)$, Fig. 8(c), and finally to a constant value of $2 \%$ at the channel centre $\left(148 \leq x^{+} \leq 150\right)$ in Fig. $8(\mathrm{~d})$. These results are in qualitatively good agreement with those of earlier studies ${ }^{8,14-16,22}$ which used the Jeffery ${ }^{25}$ equation for the torque exerted on a fibre, Eqs. (21)-(23), and which showed that particle orientation 

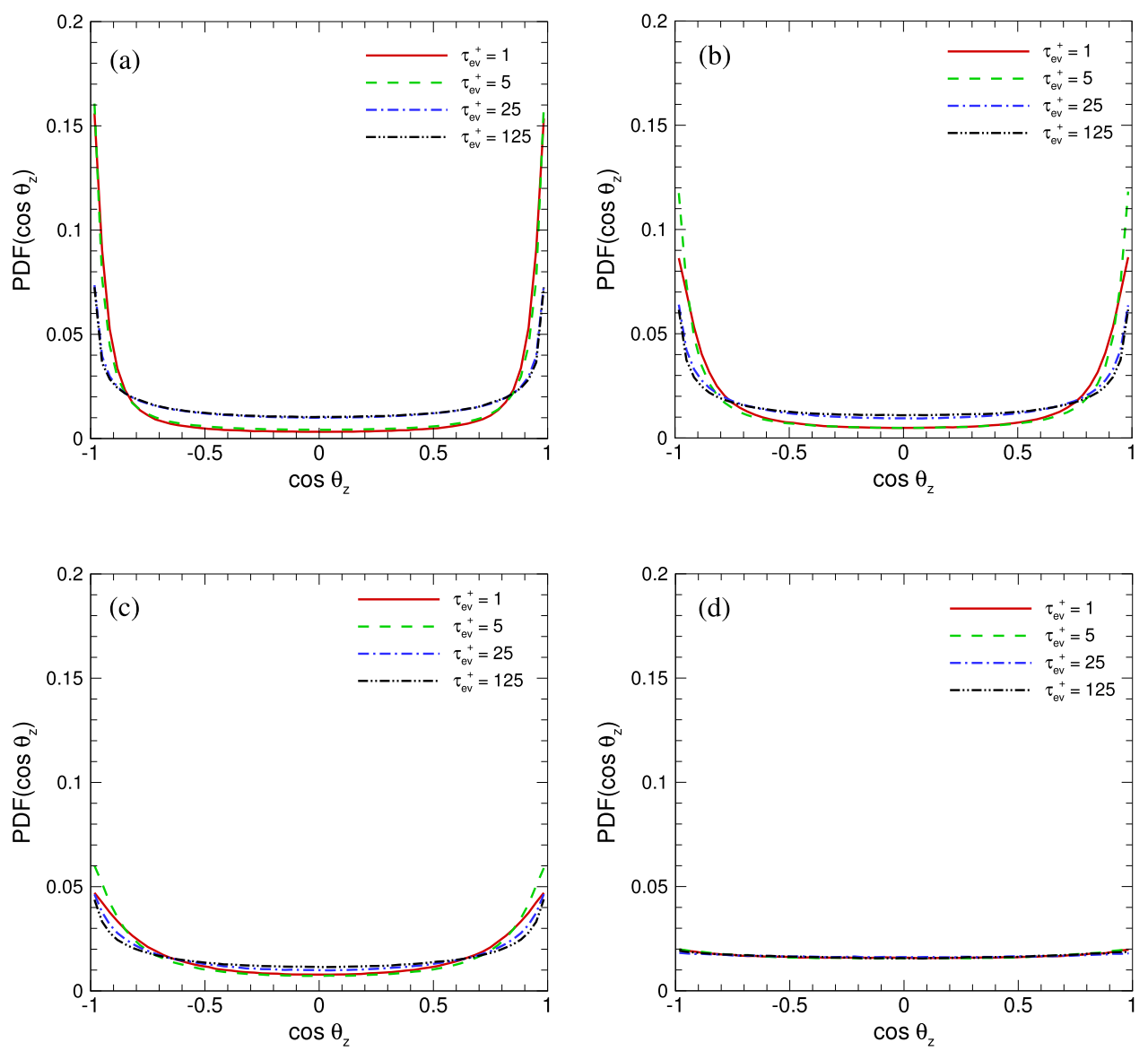

FIG. 9. Effect of channel location, $x^{+}$, and particle inertia, $\tau_{e v}^{+}$, on the probability density function of the direction cosine, $\operatorname{PDF}\left(\cos \theta_{z}\right)$, of the angle $\theta_{z}$ between the fibre principal axis, $z^{\prime}$, and the global $z$-axis at (a) $3 \leq x^{+} \leq 5$, (b) $7 \leq x^{+} \leq 9$, (c) $14 \leq x^{+} \leq 16$, and (d) $148 \leq x^{+} \leq 150$ with series representing $\tau_{e v}^{+}=(1,5,25,125)$ particles with a fixed aspect ratio, $\lambda=30$.

is correlated with the local velocity gradient, and that the alignment depends strongly on particle shape while the rotation rate is influenced by the alignment. The results demonstrate that the velocity gradient inherent in a boundary layer flow can introduce a variety of orientation states, ranging from highly aligned in the near-wall region to random orientations in the core of the flow, with the present predictions confirming and complementing previous observations, as well as extending the simulation parameter matrix. The simulations also predict a decreasing alignment of the fibres with the flow direction as the fibre size increases from $\tau_{e v}^{+}=1$ to 125, as shown in Fig. 9. Fast moving fibres with smaller inertia thus tend to align more with the flow than the slower moving fibres with larger inertia.

The PDF $\left(\cos \theta_{z}\right)$ peak at -1 and 1 means the fibres are parallel to the flow direction ( $z$-axis) with an increasing probability of parallel alignment with that direction seen for the faster moving fibres. However, the height of the peak of the PDF $\left(\cos \theta_{z}\right)$ for the fibre with relaxation time $\tau_{e v}^{+}=5$ in Figs. 9(a)-9(c) is greater than that of the fastest moving fibre with $\tau_{e v}^{+}=1$. This is because at $\tau_{e v}^{+}=1$ the shape characteristics are not as pronounced as for the $\tau_{e v}^{+}=5$ case. The highest degree of alignment with the flow direction was obtained for the $\tau_{e v}^{+}=5$ fibre when sampled in the viscous sub-layer region, Fig. 9(a).

Overall, the degree of alignment is seen to be a function of the fibre aspect ratio, the fibre inertia, and the fluid velocity gradient. The minima of the PDF around $\cos \theta_{z}=0$ are close to zero in the viscous sub-layer region in Figs. 8(a) and 9(a), showing that the fibres are unlikely to align orthogonal to the flow direction very close to the wall, irrespective of the fibre inertia and aspect ratio. The reason for this behaviour has been interpreted as a combined effect of the influence of 
the mean shear and the fluid velocity fluctuations. ${ }^{14}$ The results of Figs. 8 and 9 also reveal that the preferential orientation in the streamwise direction is most pronounced for high aspect ratios $(\lambda=50)$ and modest fibre inertia $\left(\tau_{e v}^{+}=1\right)$. The results in both figures also demonstrate that fibre alignment with the streamwise direction is strongly correlated with the fluid velocity gradient, with $\operatorname{PDF}\left(\cos \theta_{z}\right)$ decreasing by approximately half from the near-wall region to the central region of the channel where the alignment becomes weak. This finding is consistent with the qualitative observations made in regards to Fig. 4 and Fig. 7 where the fibres are seen to show strong alignment to the flow direction near the wall and at the same time no significant alignment in the core region.

Figures 10 and 11 show fibre orientation with respect to the spanwise direction, $y$ - axis, where $\theta_{y}$ represents the angle between the fibre orientation and that axis. The probability density function of the cosine of the angle $\theta_{y}$ between the fibre principal axis, $z^{\prime}$, and the spanwise direction, $y$-axis, is plotted in Fig. 10 for fibres with a fixed inertia, $\tau_{e v}^{+}=25$, and varying aspect ratio, $\lambda=1.001$, 3, 10, 30, and 50, and in Fig. 11 for fibres with a fixed aspect ratio, $\lambda=30$, and varying inertia, $\tau_{e v}^{+}=1,5,25$, and 125 . As seen for the fibre orientation with respect to the streamwise direction in Figs. 8(a)-8(c) and 9(a)-9(c), the near-spherical particles ( $\lambda=1.001)$ in Figs. 10 and 11 do not show any preferential alignment with the spanwise direction, as expected from the lack of any anisotropy in their shape. Similar to the results of Figs. 8(d) and 9(d) for fibres sampled in the channel core region, the results for all fibres, irrespective of their aspect ratio, $\lambda$, and relaxation time, $\tau_{e v}^{+}$, sampled in this region in Figs. 10(d) and 11(d) show no significant preferential alignment with respect to the spanwise direction, again as expected due to the isotropy of the local turbulence and the small
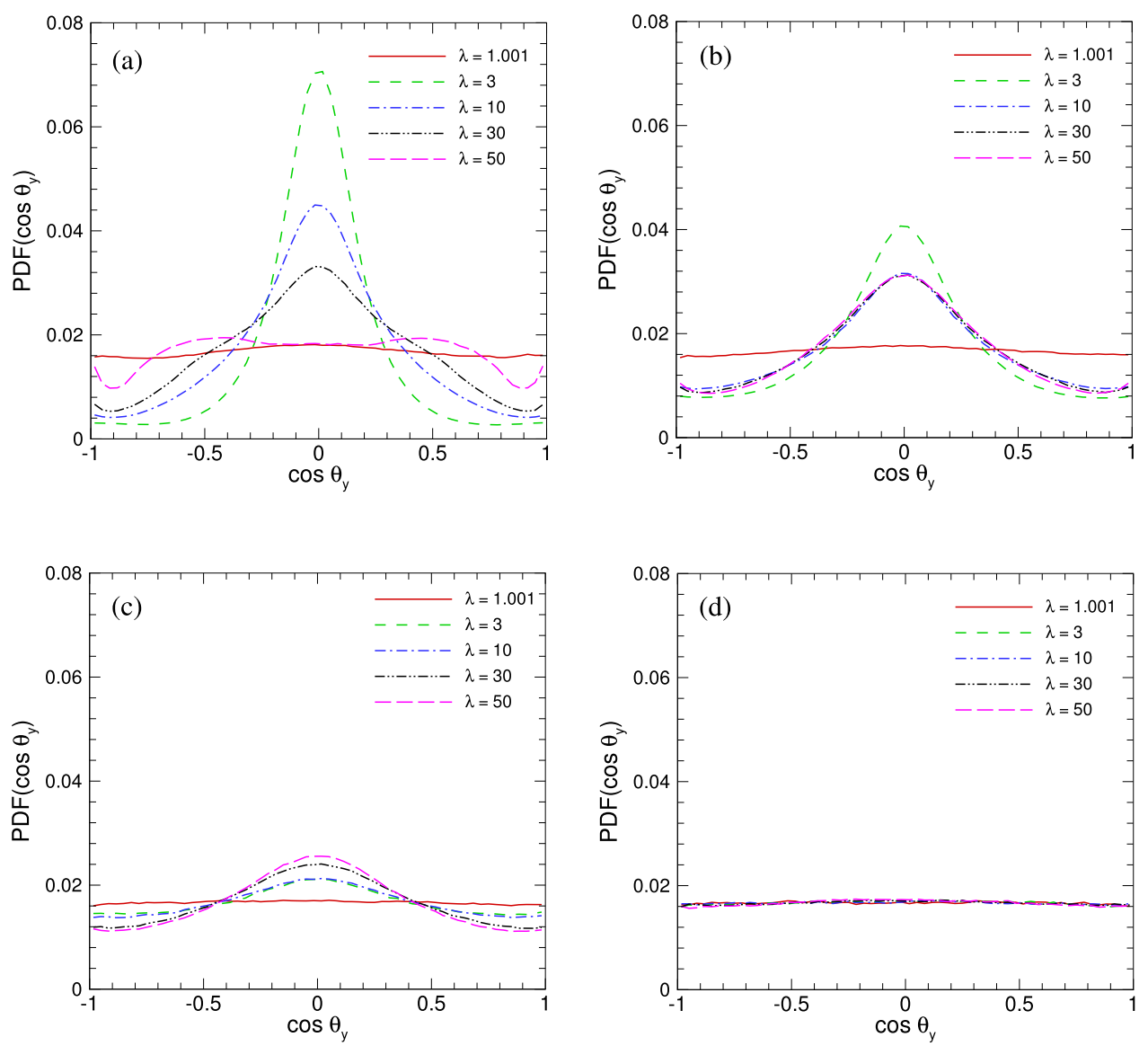

FIG. 10. Effect of channel location, $x^{+}$, and particle aspect ratio, $\lambda$, on the probability density function of the direction cosine, $\operatorname{PDF}\left(\cos \theta_{y}\right)$, of the angle $\theta_{y}$ between the fibre principal axis, $z^{\prime}$, and the global $y$-axis at: (a) $3 \leq x^{+} \leq 5$, (b) $7 \leq x^{+} \leq 9$, (c) $14 \leq x^{+} \leq 16$, and (d) $148 \leq x^{+} \leq 150$ with series representing $\lambda=(1.001,3,10,30,50)$ particles with a fixed particle inertia, $\tau_{e v}^{+}=25$. 

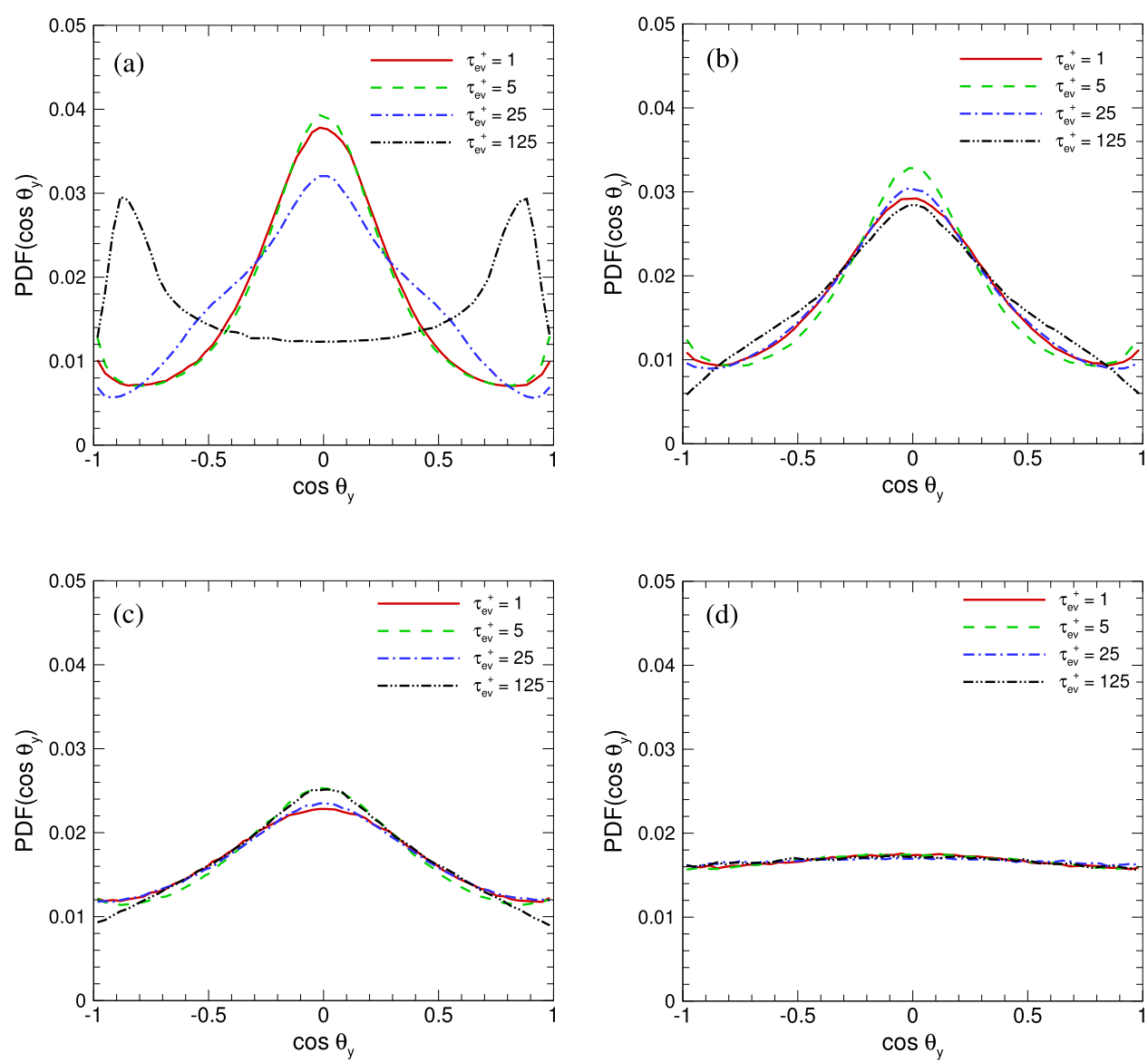

FIG. 11. Effect of channel location, $x^{+}$, and particle inertia, $\tau_{e v}^{+}$, on the probability density function of the direction cosine, $\operatorname{PDF}\left(\cos \theta_{y}\right)$, of the angle $\theta_{y}$ between the fibre principal axis, $z^{\prime}$, and the global $y$-axis at: (a) $3 \leq x^{+} \leq 5$, (b) $7 \leq x^{+} \leq 9$, (c) $14 \leq x^{+} \leq 16$, and (d) $148 \leq x^{+} \leq 150$ with series representing $\tau_{e v}^{+}=(1,5,25,125)$ particles with a fixed aspect ratio, $\lambda=30$.

velocity gradient in this region. The $\mathrm{PDF}\left(\cos \theta_{y}\right)$ peaking at $\cos \theta_{y}=0$ away from this region shows that elongated fibres $(\lambda \gg 1)$ sampled in the viscous sub-layer and buffer regions are most likely to be orthogonal to the spanwise direction, as shown in Figs. 10(a)-10(c). A decreasing probability of orthogonal alignment with the spanwise direction of the flow is seen for larger aspect ratio fibres sampled in the viscous sub-layer, as shown in Fig. 10(a). Interestingly, the most elongated fibre, $\lambda=50$, shows peaks not at $\cos \theta_{y}=0$ but at $\cos \theta_{y}=-0.5$ and $\cos \theta_{y}=0.5$ due to lateral tilting when in close proximity to the wall. The orthogonal alignment with the spanwise direction continues, but to a reduced degree, for fibres sampled in the first buffer region location of $7 \leq x^{+} \leq 9$, as shown in Fig. 10(b). Moving towards the channel centre, the velocity gradient vanishes and the distribution of the fibres' orientation with respect to the spanwise direction appears to vary weakly with aspect ratio for $\lambda \gg 1$. The fibres with larger aspect ratios exhibit a higher probability of orthogonal alignment with the spanwise direction, as seen in Fig. 10(c) where the fibre with $\lambda=50$ has the highest value of the PDF at $\cos \theta_{y}=0$.

Considering the results of Fig. 11(a), the orientation of the fibres with respect to the $y$-axis at the near-wall sampling location indicates that inertia influences orientation differently, depending on the specific value of the inertia. In this region, sufficiently inertial fibres, with $\tau_{e v}^{+}=125$, are aligned in planes perpendicular to the flow direction, i.e., in the spanwise direction, as the $\operatorname{PDF}\left(\theta_{y}\right)$ for these fibres shows two maxima at $\cos \theta_{y} \sim-1$ and $\cos \theta_{y} \sim 1$, and a minimum at $\cos \theta_{y}=0$, again due to lateral tilting when in close proximity to the wall. As the inertia increases to $\tau_{e v}^{+}=125$, therefore, the length of the fibre semi-major axis increases for a fixed aspect ratio; hence, an additional effect of the intersection of the fibre leading edge with the wall comes into play and causes 

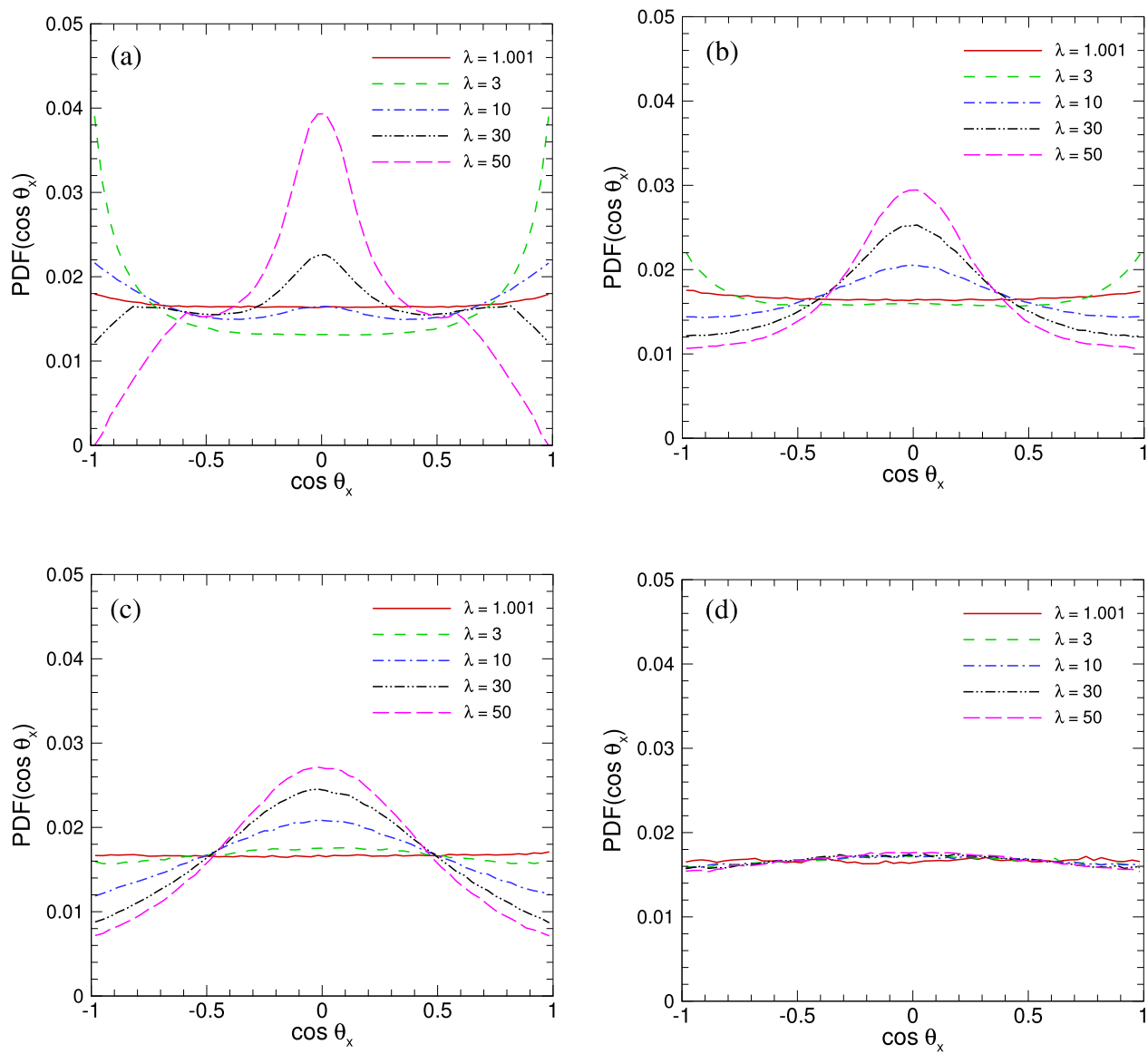

FIG. 12. Effect of channel location, $x^{+}$, and particle aspect ratio, $\lambda$, on the probability density function of the direction cosine, $\operatorname{PDF}\left(\cos \theta_{x}\right)$, of the angle $\theta_{x}$ between the fibre principal axis, $z^{\prime}$, and the global $x$-axis at (a) $3 \leq x^{+} \leq 5$, (b) $7 \leq x^{+} \leq 9$, (c) $14 \leq x^{+} \leq 16$, and (d) $148 \leq x^{+} \leq 150$ with series representing $\lambda=(1.001,3,10,30,50)$ particles with a fixed particle inertia, $\tau_{e v}^{+}=25$.

the orientation of the largest fibres to deviate from that of the smaller fibres. Hence the appearance of the two peaks close to the wall in Fig. 11(a) for the fibre with the largest inertia. Comparing the value of the peak of the PDF $\left(\cos \theta_{y}\right)$ in Fig. 11(a) with those of the same fibres in Fig. 9(a), and with the fibre behaviour shown in Fig. 7, it can be seen that the very large fibres show preferential alignment with the flow direction, $z$-axis, and the spanwise direction $y$-axis in the near-wall region. The degree of alignment is higher in the flow direction than in the spanwise direction as the peak of the $\operatorname{PDF}\left(\cos \theta_{z}\right)$ is approximately 0.08 compared to approximately 0.03 obtained for the peak of the $\operatorname{PDF}\left(\cos \theta_{y}\right)$. With moderate fibre inertia, $\tau_{e v}^{+} \leq 25$, the $\operatorname{PDF}\left(\cos \theta_{y}\right)$ shows a peak at $\cos \theta_{y}=0$ indicating that the fibres are aligned perpendicular to the $y$ - axis, with the degree of such alignment increasing as the fibre inertia decreases towards $\tau_{e v}^{+}=1$. This behaviour is also observed for all the fibres sampled in the buffer region, as shown in Figs. 11(b) and 11(c). In this case, close to the wall in Fig. 11(a), the fibre Reynolds number, $R e_{p}$, is high because of the high slip velocity, and inertial effects associated with the large fibre Reynolds number become dominant and contribute to the fibres aligning with the direction of the flow, and hence perpendicular to the $y$-axis. This is evident as the probability of this alignment reduces as the particle location moves away from the wall towards the centre of the channel, i.e., as $R e_{p}$ decreases.

The fibre orientation with respect to the flow gradient direction, $x$-axis, at four wall-normal locations shown in Figs. 12 and 13 is consistent with that obtained with respect to the spanwise direction in Figs. 10 and 11, and corroborates the results obtained with respect to the streamwise direction in Figs. 8 and 9. Again, the results establish that the orientation distribution is strongly 

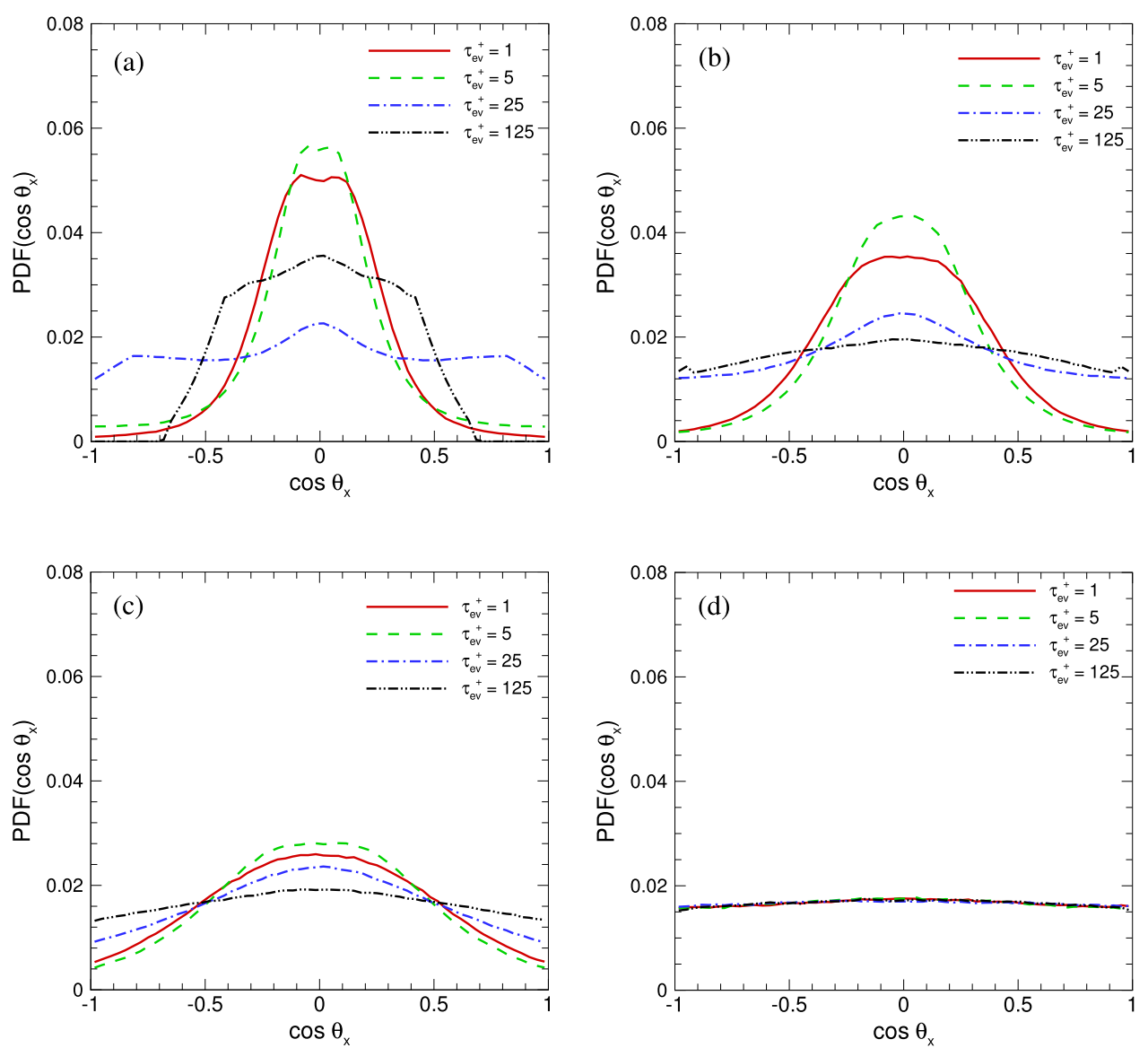

FIG. 13. Effect of channel location, $x^{+}$, and particle inertia, $\tau_{e v}^{+}$, on the probability density function of the direction cosine, $\operatorname{PDF}\left(\cos \theta_{x}\right)$, of the angle $\theta_{x}$ between the fibre principal axis, $z^{\prime}$, and the global $x$-axis at (a) $3 \leq x^{+} \leq 5$, (b) $7 \leq x^{+} \leq 9$, (c) $14 \leq x^{+} \leq 16$, and (d) $148 \leq x^{+} \leq 150$ with series representing $\tau_{e v}^{+}=(1,5,25,125)$ particles with a fixed aspect ratio, $\lambda=30$.

influenced by the flow velocity gradient as there is a significant difference in the orientation in the near-wall region when compared to the near-isotropic distribution far-away from the wall. The angle $\left(\theta_{x}\right)$ that define the fibres' preferential alignment with respect to the wall-normal direction is expected to be complementary to that $\left(\theta_{z}\right)$ which defines their alignment with respect to the streamwise direction presented in Figs. 8 and 9. This is because the fibres that are predominantly parallel to the streamwise direction, e.g., $\lambda=50$, can, at the same time, only be predominantly perpendicular to the wall-normal direction. Hence, in Fig. 12(a), sufficiently elongated fibres $(\lambda=30$ and 50) which are strongly aligned parallel to the streamwise axis in Fig. 8(a) are strongly aligned perpendicular to the wall-normal axis, with a decreasing degree of alignment as the elongation reduces to that of a sphere. The fibres with aspect ratio $\lambda=10$ show no preferential alignment with respect to the $x$-axis, and a further decrease in the aspect ratio reveals a change in the orientation from strong perpendicular alignment for the elongated fibres $(\lambda=30$ and 50) to a weak alignment parallel with the $x$-axis for the least elongated fibres $(\lambda=1.001$ and 3$)$. As we move away from the wall, Figs. 12(b) and 12(c), the alignment becomes weaker but maintains similar trends to those observed in the near-wall region in Fig. 12(a). All fibres, however, remain randomly orientated in the channel centre, as attested by the probability density function $\mathrm{PDF}\left(\cos \theta_{x}\right)$ which has a uniform distribution for all fibre aspect ratios in Fig. 12(d) and for all fibre inertias in Fig. 13(d).

For all fibre inertia in Figs. 13(a)-13(c), the peak of the PDF at $\cos \theta_{x} \sim 0$ indicates a strong preferential alignment perpendicular to the wall-normal direction. In general, fibres with low inertia in Fig. 13(a) show the highest probability, an indication that alignment parallel to the wall-normal 
direction ( $x$-axis) increases with a decrease in their inertia. In addition, the degree of alignment decreases as the magnitude of the flow velocity gradient decreases from locations near the wall to those at the channel centre. Again, near the centre of the channel, the alignment disappears and the fibre orientation becomes random. This trend is consistent with the alignment noted with the streamwise direction in Fig. 8 where all shapes of particle were found to show no preferential orientation.

\section{CONCLUSIONS}

In this paper, we have presented a CFD alternative to the widely used Stokesian dynamics method for simulating the translational and rotational dynamics of non-spherical particles in turbulent flow. The developed method combines an Eulerian-Lagrangian technique and the Eulerian rotation equation, in the context of large eddy simulation. The method accounts for inertial particles with large Reynolds number in a fully developed turbulent channel flow. The method adopted is essentially a modification of the method of Yin et al. ${ }^{40}$ developed for cylindrical particle dynamics in a simple non-uniform flow. The present simulations adopted empirical drag coefficient correlations and invoked the fibre projected area, $A_{D}$, and the incidence angle, $\alpha_{i}$, to account for the dependence on the particle dynamic orientation. The particle incidence angle is obtained from the particle slip-velocity and fibre Euler quaternions. The linear Jeffery ${ }^{25}$ torque was adopted in lieu of the Yin et al.,$^{40}$ torque due to the resistance to rotational motion. More importantly, an accurate fibre-wall contact model was implemented to ensure that near-wall fibre behaviour was captured. Overall, the method and results reported are an extension of previous work by Zhang et al. ${ }^{14}$ and Mortensen et al., ${ }^{15,16}$ where only modest sizes of fibre $\left(\tau_{e v}^{+} \leq 30\right)$ and moderate elongations $(\lambda \leq 30)$ were investigated, and by Marchioli et al. ${ }^{8}$ where a Stokesian, or creeping flow, regime was assumed. A wider fibre parameter space of $\tau_{e v}^{+}=1,5,25$, and 125 and $\lambda=1.001,3,10,30$, and 50 was reported in the present paper.

The overall model was applied in a one-way coupled large eddy simulation of a turbulent channel flow at shear Reynolds number $R e_{\tau}=150$. As noted, a wider fibre parameter space than in previous works was investigated, with simulations used to determine the fibres' orientational and concentration behaviour at different wall-normal positions. The streaky nature of the flow in the near-wall region was confirmed, with elongated regions of low-speed fluid alternating with regions of fast-moving fluid. The fibres were demonstrated to preferentially accumulate in the low speed streaks due to ejection and sweep events, with their orientation strongly aligning with the direction of flow. The results confirm the findings of previous, more limited studies of fibre alignment, in that the fibres were found to be more aligned with the flow direction at positions near to a wall where the velocity gradient is highest. The degree of alignment with the flow direction becomes weaker in moving away from the wall region and subsequently vanishes at the channel centre where local velocity gradients are low and turbulence near-isotropic. As anticipated, therefore, near-spherical particles with aspect ratio $\lambda=1.001$, irrespective of their size $\tau_{e v}^{+}$, and all fibres, irrespective of size and shape $\left(\tau_{e v}^{+}, \lambda\right)$, sampled at the channel centre showed a randomised, or zero, alignment with the fixed channel axes, $\mathbf{x}=[x, y, z]$.

The main parameters governing fibre orientation distribution with respect to the fixed axis, $\mathbf{x}=[x, y, z]$, were found to be the flow velocity gradient, fibre inertia, and fibre aspect ratio. The shortest near-spherical particles $(\lambda=1.001)$ were found to have no preferential alignment with any of the three co-ordinate directions, while longer fibres $(\lambda \gg 1)$ tend to adopt an orientation parallel only to the flow direction. Values of the probability density function, $\operatorname{PDF}\left(\cos \theta_{z}\right)$, of the direction cosine of the angle $\theta_{z}$ between the fibre principal axis, $z^{\prime}$, and the streamwise direction, $z$-axis, demonstrated that fibre alignment with the flow direction decreases as fibre inertia decreases, and as the shape of the fibre approaches spherical. With respect to the vorticity and velocity gradient directions, and for the range of parameters investigated, the fibres generally show orientations perpendicular to those directions. 
Overall, the numerical results demonstrate that the simulation approach represents a useful method for investigating the interaction between fibres and complex fluid flow patterns in a relatively simple flow. More specifically, the modification of Jeffery's equation to account for inertial effects by the addition of empirical correlations and torques due to rotation has been shown to be adequate to model inertial fibre motion in turbulent flow. Knowledge of the fibre orientation and local concentration will be used in future to investigate fibre interactions that can ultimately lead to their agglomeration.

\section{ACKNOWLEDGMENTS}

The authors wish to thank the Engineering and Physical Sciences Research Council for their financial support of the work reported in this paper under EPSRC Grant No. EP/I003010/1, "Computational Modelling for Advanced Nuclear Power Plants."

${ }^{1}$ D. L. Koch and G. Subramanian, "Collective hydrodynamics of swimming microorganisms: Living fluids," Annu. Rev. Fluid Mech. 43, 637 (2011).

${ }^{2}$ C. R. Meyer, M. L. Byron, and E. A. Variano, "Rotational diffusion of particles in turbulence," Limnol. Oceanogr.: Fluids Environ. 3, 89 (2013).

${ }^{3}$ F. Lundell, L. D. Söderberg, and P. H. Alfredsson, "Fluid mechanics of papermaking," Annu. Rev. Fluid Mech. 43, 195 (2011).

${ }^{4}$ R. F. Hazelton, "Characteristics of fuel crud and its impact on storage, handling, and shipment of spent fuel," PNL-6273, UC-85, DE88 000914, Pacific Northwest Laboratory, Richland, WA, 1987.

${ }^{5}$ M. B. Pinsky and A. P. Khain, "Some effects of cloud turbulence on water-ice and ice-ice collisions," Atmos. Res. 47-48, 69 (1998)

${ }^{6}$ K. K. Zeming, S. Ranjan, and Y. Zhang, "Rotational separation of non-spherical bioparticles using I-shaped pillar arrays in a microfluidic device," Nat. Commun. 4, 1625 (2013).

${ }^{7}$ K. Zhou, J. Lin, and T. L. Chan, "Solution of three-dimensional fiber orientation in two-dimensional fiber suspension flows," Phys. Fluids 19, 113309 (2007).

${ }^{8}$ C. Marchioli, M. Fantoni, and A. Soldati, "Orientation, distribution, and deposition of elongated, inertial fibers in turbulent channel flow," Phys. Fluids 22, 033301 (2010).

${ }^{9}$ S. Mueller, E. W. Llewellin, and H. M. Mader, “The rheology of suspensions of solid particles,” Proc. R. Soc. A 466, 1201 (2010).

10 J. J. J. Gillissen, B. J. Boersma, P. H. Mortensen, and H. I. Andersson, “Fibre-induced drag reduction,” J. Fluid Mech. 602, 209 (2008).

${ }^{11}$ H. I. Andersson, L. Zhao, and M. Barri, “Torque-coupling and particle-turbulence interactions,” J. Fluid Mech. 696, 319 (2012).

12 J.-Z. Lin, K. Sun, and J. Lin, "Distribution of orientations in fibre suspension flowing in a turbulent boundary layer," Chin. Phys. Lett. 22, 3111 (2005).

${ }^{13}$ D. L. Koch and E. S. G. Shaqfeh, “The instability of a dispersion of sedimenting spheroids,” J. Fluid Mech. 209, 521 (1989).

${ }^{14}$ H. Zhang, G. Ahmadi, F.-G. Fan, and J. B. McLaughlin, "Ellipsoidal particles transport and deposition in turbulent channel flows," Int. J. Multiphase Flow 27, 971 (2001).

${ }^{15}$ P. H. Mortensen, H. I. Andersson, J. J. J. Gillissen, and B. J. Boersma, "Dynamics of prolate ellipsoidal particles in a turbulent channel flow," Phys. Fluids 20, 093302 (2008).

${ }^{16}$ P. H. Mortensen, H. I. Andersson, J. J. J. Gillissen, and B. J. Boersma, "On the orientation of ellipsoidal particles in a turbulent shear flow,” Int. J. Multiphase Flow 34, 678 (2008).

${ }^{17}$ L. H. Zhao, C. Marchioli, and H. I. Andersson, "Stokes number effects on particle slip velocity in wall-bounded turbulence and implications for dispersion models," Phys. Fluids 24, 021705 (2012).

${ }^{18}$ L. Zhao, C. Marchioli, and H. I. Andersson, "Slip velocity of rigid fibers in turbulent channel flow,” Phys. Fluids 26, 063302 (2014).

${ }^{19}$ C. A. Stover, D. L. Koch, and C. Cohen, "Observations of fibre orientation in simple shear flow of semi-dilute suspensions," J. Fluid Mech. 238, 277 (1992).

${ }^{20}$ A. Carlsson, L. D. Söderberg, and F. Lundell, "Fiber orientation control related to papermaking,” J. Fluids Eng. 129, 457 (2006).

${ }^{21}$ S. Dearing, M. Campolo, A. Capone, and A. Soldati, "Phase discrimination and object fitting to measure fibers distribution and orientation in turbulent pipe flows," Exp. Fluids 54, 1419 (2012).

${ }^{22}$ S. Parsa, E. Calzavarini, F. Toschi, and G. A. Voth, "Rotation rate of rods in turbulent fluid flow," Phys. Rev. Lett. 109, 134501 (2012).

${ }^{23}$ K. O. Hakansson, M. Kvick, F. Lundell, L. Prahl Wittberg, and L. D. Söderberg, "Measurement of width and intensity of particle streaks in turbulent flows," Exp. Fluids 54, 1555 (2013).

${ }^{24}$ G. Qi, G. J. Nathan, and T. C. W. Lau, "Velocity and orientation distributions of fibrous particles in the near-field of a turbulent jet," Powder Technol. 276, 10 (2015).

${ }^{25}$ G. B. Jeffery, "The motion of ellipsoidal particles immersed in a viscous fluid," Proc. R. Soc. A 102, 161 (1922).

${ }^{26}$ H. Brenner, "The Stokes resistance of an arbitrary particle," Chem. Eng. Sci. 18, 1 (1963).

${ }^{27}$ H. Brenner, "The Stokes resistance of an arbitrary particle-IV. Arbitrary fields of flow," Chem. Eng. Sci. 19, 703 (1964). 
${ }^{28}$ M. Mandø and L. Rosendahl, "On the motion of non-spherical particles at high Reynolds number,” Powder Technol. 202, 1 (2010).

${ }^{29}$ G. Bellani, M. L. Byron, A. G. Collignon, C. R. Meyer, and E. A. Variano, "Shape effects on turbulent modulation by large nearly neutrally buoyant particles," J. Fluid Mech. 712, 41 (2012).

${ }^{30}$ M. Zastawny, G. Mallouppas, F. Zhao, and B. van Wachem, "Derivation of drag and lift force and torque coefficients for non-spherical particles in flows," Int. J. Multiphase Flow 39, 227 (2012).

${ }^{31}$ B. van Wachem, M. Zastawny, F. Zhao, and G. Mallouppas, "Modelling of gas-solid turbulent channel flow with nonspherical particles with large Stokes numbers," Int. J. Multiphase Flow 68, 80 (2015).

${ }^{32}$ W. Mao and A. Alexeev, "Motion of spheroid particles in shear flow with inertia," J. Fluid Mech. 749, 145 (2014)

${ }^{33}$ T. Rosén, F. Lundell, and C. K. Aidun, "Effect of fluid inertia on the dynamics and scaling of neutrally buoyant particles in shear flow," J. Fluid Mech. 738, 563 (2014).

${ }^{34}$ T. Rosén, M. Do-Quang, C. K. Aidun, and F. Lundell, "The dynamical states of a prolate spheroidal particle suspended in shear flow as a consequence of particle and fluid inertia," J. Fluid Mech. 771, 115 (2015).

35 T. Rosén, M. Do-Quang, C. K. Aidun, and F. Lundell, "Effect of fluid and particle inertia on the rotation of an oblate spheroidal particle suspended in linear shear flow," Phys. Rev. E 91, 053017 (2015).

${ }^{36}$ H. Huang, X. Yang, M. Krafczyk, and X.-Y. Lu, "Rotation of spheroidal particles in Couette flows," J. Fluid Mech. 692, 369 (2012).

${ }^{37}$ D. Qi and L. Luo, "Rotational and orientational behaviour of three-dimensional spheroidal particles in Couette flows," J. Fluid Mech. 477, 201 (2003).

${ }^{38}$ M. Do-Quang, G. Amberg, G. Brethouwer, and A. V. Johansson, "Simulation of finite-size fibers in turbulent channel flows," Phys. Rev. E 89, 013006 (2014).

${ }^{39}$ D. O. Njobuenwu and M. Fairweather, "Dynamics of single, non-spherical ellipsoidal particles in a turbulent channel flow," Chem. Eng. Sci. 123, 265 (2015).

${ }^{40}$ C. Yin, L. Rosendahl, S. Knudsen Kær, and H. Sørensen, "Modelling the motion of cylindrical particles in a nonuniform flow," Chem. Eng. Sci. 58, 3489 (2003).

${ }^{41}$ C. Yin, L. Rosendahl, S. K. Kær, and T. J. Condra, "Use of numerical modeling in design for co-firing biomass in wall-fired burners," Chem. Eng. Sci. 59, 3281 (2004).

42 D. O. Njobuenwu and M. Fairweather, "Effect of shape on inertial particle dynamics in a channel flow," Flow, Turbul. Combust. 92, 83 (2014).

${ }^{43}$ S. Laín and M. Sommerfeld, "A study of the pneumatic conveying of non-spherical particles in a turbulent horizontal channel flow,” Braz. J. Chem. Eng. 24, 535 (2007).

${ }^{44}$ S. R. Gubba, D. B. Ingham, K. J. Larsen, L. Ma, M. Pourkashanian, X. Qian, A. Williams, and Y. Yan, "Investigations of the transportation characteristics of biomass fuel particles in a horizontal pipeline through CFD modelling and experimental measurement," Biomass Bioenergy 46, 492 (2012).

45 U. Piomelli and J. Liu, "Large-eddy simulation of rotating channel flows using a localized dynamic model," Phys. Fluids 7, 839 (1995)

46 J. Smagorinsky, "General circulation experiments with the primitive equations," Mon. Weather Rev. 91, 99 (1963).

${ }^{47}$ M. Bini and W. P. Jones, "Large-eddy simulation of particle-laden turbulent flows," J. Fluid Mech. 614, 207 (2008).

${ }^{48}$ M. R. Maxey and J. J. Riley, "Equation of motion for a small rigid sphere in a nonuniform flow," Phys. Fluids 26, 883 (1983)

${ }^{49}$ M. Bini and W. P. Jones, "Particle acceleration in turbulent flows: A class of nonlinear stochastic models for intermittency," Phys. Fluids 19, 035104 (2007).

${ }^{50}$ G. Sardina, P. Schlatter, L. Brandt, F. Picano, and C. M. Casciola, "Wall accumulation and spatial localization in particleladen wall flows," J. Fluid Mech. 699, 50 (2012).

${ }^{51}$ G. H. Ganser, "A rational approach to drag prediction of spherical and nonspherical particles," Powder Technol. 77, 143 (1993).

${ }^{52}$ F.-G. Fan and G. Ahmadi, "A sublayer model for wall deposition of ellipsoidal particles in turbulent streams," J. Aerosol Sci. 26, 813 (1995).

${ }^{53}$ M.-C. Kim and C. Klapperich, "A new method for simulating the motion of individual ellipsoidal bacteria in microfluidic devices," Lab Chip 10, 2464 (2010).

${ }^{54}$ L. di Mare and W. P. Jones, "LES of turbulent flow past a swept fence," Int. J. Heat Fluid Flow 24, 606 (2003).

${ }^{55}$ L. Gamet, F. Ducros, F. Nicoud, and T. Poinsot, "Compact finite difference schemes on non-uniform meshes. Application to direct numerical simulations of compressible flows," Int. J. Numer. Methods Fluids 29, 159 (1999).

${ }^{56}$ C. Marchioli, A. Soldati, J. G. M. Kuerten, B. Arcen, A. Tanière, G. Goldensoph, K. D. Squires, M. F. Cargnelutti, and L. M. Portela, "Statistics of particle dispersion in direct numerical simulations of wall-bounded turbulence: Results of an international collaborative benchmark test," Int. J. Multiphase Flow 34, 879 (2008).

${ }^{57}$ N. R. Challabotla, L. Zhao, and H. I. Andersson, "Orientation and rotation of inertial disk particles in wall turbulence," J. Fluid Mech. 766, R2 (2015).

${ }^{58}$ F.-G. Fan and G. Ahmadi, "Wall deposition of small ellipsoids from turbulent air flows-A Brownian dynamics simulation," J. Aerosol Sci. 31, 1205 (2000).

${ }^{59}$ C. Narayanan, D. Lakehal, L. Botto, and A. Soldati, "Mechanisms of particle deposition in a fully developed turbulent open channel flow," Phys. Fluids 15, 763 (2003).

${ }^{60}$ D. O. Njobuenwu and M. Fairweather, "Quadrant and conditional analyses of non-spherical particle segregation in a turbulent channel flow," Procedia Eng. 102, 123 (2015).

${ }^{61}$ J. R. Fessler, J. D. Kulick, and J. K. Eaton, "Preferential concentration of heavy particles in a turbulent channel flow," Phys. Fluids 6, 3742 (1994).

${ }^{62}$ C. Marchioli and A. Soldati, "Mechanisms for particle transfer and segregation in a turbulent boundary layer," J. Fluid Mech. 468, 283 (2002).

${ }^{63}$ D. Vincenzi, "Orientation of non-spherical particles in an axisymmetric random flow," J. Fluid Mech. 719, 465 (2013). 